\title{
CaBER vs ROJER-Different time scales for the thinning of a weakly elastic jet
}

Wouter Mathues, Susanna Formenti, Claire Mcllroy, Oliver G. Harlen, and Christian Clasen

Citation: Journal of Rheology 62, 1135 (2018); doi: 10.1122/1.5021834

View online: https://doi.org/10.1122/1.5021834

View Table of Contents: https://sor.scitation.org/toc/jor/62/5

Published by the The Society of Rheology

\section{ARTICLES YOU MAY BE INTERESTED IN}

Drop breakup dynamics of dilute polymer solutions: Effect of molecular weight, concentration, and viscosity Journal of Rheology 62, 1245 (2018); https://doi.org/10.1122/1.5038000

Relaxation time of dilute polymer solutions: A microfluidic approach Journal of Rheology 61, 327 (2017); https://doi.org/10.1122/1.4975933

Linear shear and nonlinear extensional rheology of unentangled supramolecular side-chain polymers Journal of Rheology 62, 1155 (2018); https://doi.org/10.1122/1.5012349

Steady-state extensional viscosity of a linear polymer solution using a differential pressure extensional rheometer on a chip

Journal of Rheology 62, 1261 (2018); https://doi.org/10.1122/1.5033499

Continuous relaxation spectra for constitutive models in medium-amplitude oscillatory shear Journal of Rheology 62, 1271 (2018); https://doi.org/10.1122/1.5025080

Sphere sedimentation in wormlike micelles: Effect of micellar relaxation spectrum and gradients in micellar extensions

Journal of Rheology 62, 1061 (2018); https://doi.org/10.1122/1.5031899

\section{Master your flow}

with the MCR Rheometer series
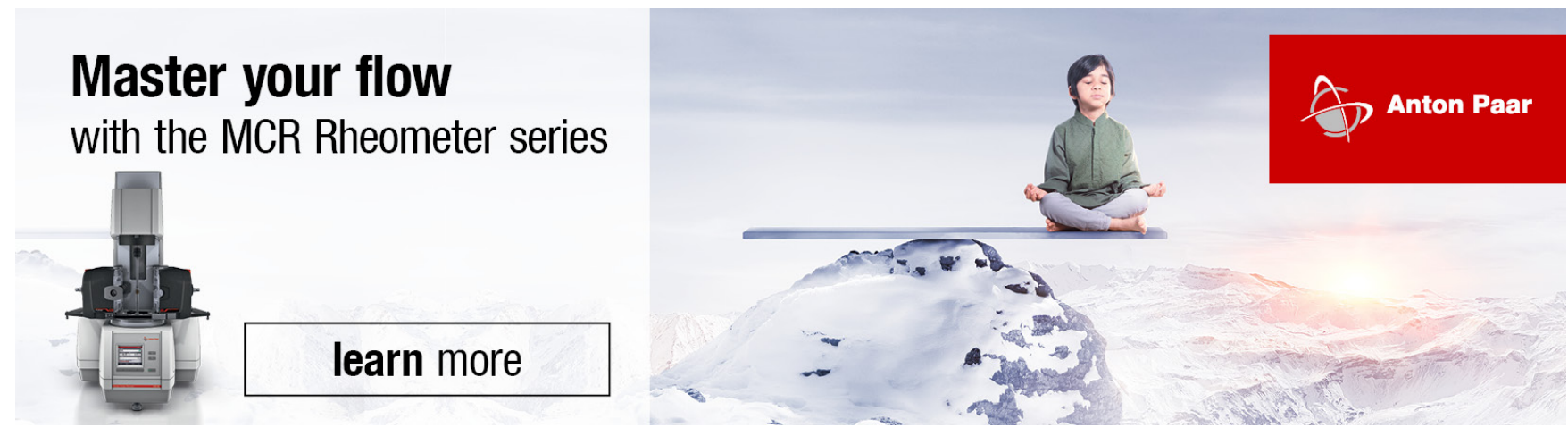


\title{
CaBER vs ROJER-Different time scales for the thinning of a weakly elastic jet
}

\author{
Wouter Mathues, ${ }^{1}$ Susanna Formenti, ${ }^{1,2}$ Claire McIlroy, ${ }^{3, a)}$ Oliver G. Harlen, ${ }^{3}$ and Christian Clasen ${ }^{1, b)}$ \\ ${ }^{1}$ Department of Chemical Engineering, KU Leuven, Celestijnenlaan $200 \mathrm{~F}, 3001$ Leuven, Belgium \\ ${ }^{2}$ Department of Chemistry, Materials and Chemical Engineering G. Natta, Politecnico di Milano, piazza Leonardo da \\ Vinci 32, 20133 Milan, Italy \\ ${ }^{3}$ Department of Applied Mathematics, University of Leeds, Woodhouse Lane, Leeds LS2 9JT, United Kingdom
}

(Received 9 January 2018; final revision received 29 June 2018; published 7 August 2018)

\begin{abstract}
In this paper, we demonstrate that the capillary thinning dynamics of a weakly viscoelastic jet follow a different timescale than a liquid bridge of the same fluid between two stationary surfaces for similar geometrical scales. The thinning in the latter case observed with capillary breakup extensional rheometry (or CaBER) follows a well established scaling of the radius with time for an elasto-capillary (EC) balance of $R \sim \exp (-t / 3 \lambda)$. However, for the thinning of the filaments between droplets in a jet, it was so far just assumed that the same scaling law holds. In this paper, we experimentally demonstrate that the jet thinning in a Rayleigh-Ohnesorge jetting extensional rheometer (or ROJER) follows a different scaling of $R \sim \exp (-t / 2 \lambda)$. This is demonstrated by a direct comparison of the thinning dynamics of weakly viscoelastic $(O h<0.01)$ aqueous solutions of polyethylene oxide in the two experimental setups, covering a wide range of jetting velocities or Weber numbers of 1-70. We demonstrate outgoing from a momentum balance that includes inertia and elasticity that this difference in scaling is due to a constant axial tension in the jet arising from the constant creation rate of new surface at the nozzle. Numerical simulations using the FENE-P model support this theory and demonstrate that in the exponential thinning regime of the jet the elastic stresses are indeed balanced by the axial tension (rather than by capillary pressure as in the EC balance regime of the CaBER experiment). It is readily shown from the reduced stress balance that this axial-elastic balance regime in the ROJER experiment leads to a faster exponential thinning, following the new scaling of $R \sim \exp (-t / 2 \lambda)$ that was experimentally observed. Furthermore, we observe both in experiment and simulation that a jet thinning does not exhibit a self-similar structure of the corner region where the thinning filament connects to the drop as it is generally observed for a filament with an axial tension decaying with the filament radius as in the CaBER. The resulting difference of 50\% in extensional relaxation time $\lambda$ extracted from ROJER experiments might require one to revisit previously reported ROJER experiments and is required for the correct evaluation of future jetting rheometry experiments. (C) 2018 The Society of Rheology.

https://doi.org/10.1122/1.5021834
\end{abstract}

\section{INTRODUCTION}

When a liquid is sent through a nozzle at sufficiently high velocity, a liquid jet is formed that spontaneously disintegrates into a series of small droplets under the influence of surface tension. The cylindrical fluid column that appears after the nozzle exit is inherently unstable due to capillarity. Sinusoidal disturbances on the jet surface with a sufficiently large wavelength reduce the surface area and are thus thermodynamically favorable as they reduce the surface energy. The amplitude of the instability grows exponentially in time with a particular growth rate that depends on the wavelength. As the jet flows downstream, the instability progressively thins and the jet disintegrates into a series of droplets with sizes that depend on the wavelength of the perturbation $[1,2]$.

Adding a small amount of flexible polymers to the liquid considerably alters the thinning dynamics of the jet [3]. Performing a linear stability analysis on a viscoelastic jet shows that the instabilities grow faster compared to a

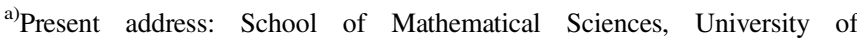
Nottingham, Nottingham NG7 2RD, United Kingdom.

b) Author to whom correspondence should be addressed; electronic mail: christian.clasen@kuleuven.be
}

Newtonian fluid with the same inertia and zero-shear viscosity $[4,5]$. However, nonlinear effects quickly dominate the breakup of these fluids. As the local radius of the jet decreases, elastic stresses grow and the jet profile consists of a series of small droplets joined by small threads, which thin slower than a purely viscous liquid [4,6,7]. This typical "beads-on-a-string" morphology is displayed in Figs. 1(a) and 1(b) for aqueous polyethylene oxide (PEO) solutions.

Viscoelastic jets are encountered in various spraying and dispensing operations [8]. For several processes, small concentrations of polymer are included in the fluid formulation to alter the atomization characteristics. Examples include high molecular weight polyisobutylene (PIB) that is used to suppress misting of numerous inflammable liquids [9], or appropriate concentrations of polymers of a desirable molecular weight that are added to inkjet printing fluids to control the droplet deposition [10]. Such polymer additions can suppress the formation of unwanted satellite drops, which arise during the capillary breakup of a Newtonian fluid and cause a reduction in the printing quality [11-14].

Throughout the jetting process, the fluid is subjected to a complex extensional deformation with rapidly varying strain rates. The breakup is driven by the capillary pressure within the thread and is resisted by inertial, viscous or elastic stresses in the fluid, depending on the fluid characteristics 
a)

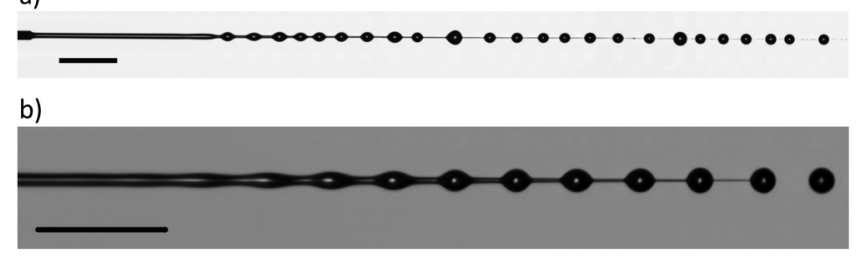

c)

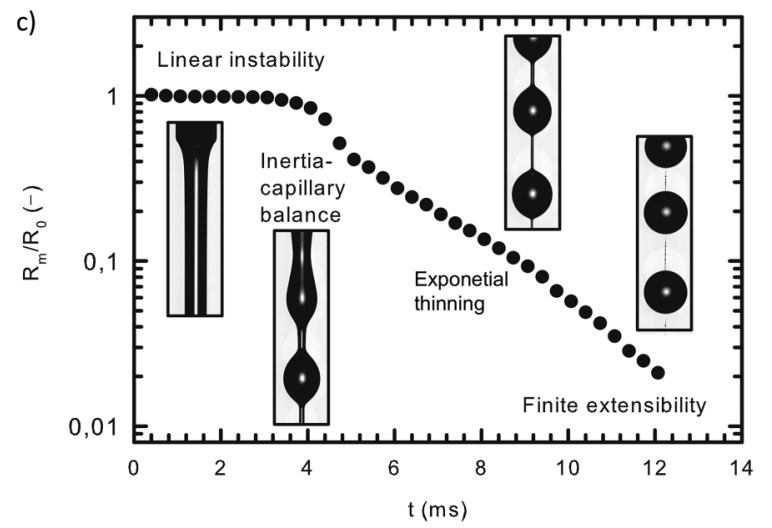

FIG. 1. (a) Image of an unexcited jet from the nozzle to breakup at approximately $30 \mathrm{~mm}$ from the nozzle for a $0.1 \mathrm{wt}$. \% PEO solution from a nozzle with an inner radius $R_{n}=75 \mu \mathrm{m}$ at $W e=4.0$. (b) Image of an excited jet for a 0.001 wt. $\%$ PEO in water/glycerol $60 / 40$ (wt. \%) solution from a nozzle with an inner radius $R_{n}=100 \mu \mathrm{m}$ at $W e=4.0$. The scale bar in both images represents $2 \mathrm{~mm}$. (c) The radius of an instability of jet (a) as a function of time. The growth of the instabilities is illustrated with a representative picture for each of the regimes.

and the deformation history. The relative importance of these resisting stresses determines the temporal evolution of the jet and can be expressed by dimensionless numbers $[8,15]$. One is the Ohnesorge number that compares the viscous and inertial effects

$$
O h=\frac{\eta}{\sqrt{\rho \gamma R}}
$$

where $\eta$ is the viscosity, $\rho$ is the density, $\gamma$ is the surface tension, and $R$ is a characteristic radius. The Ohnesorge number can be considered as the ratio between the viscous time scale $\eta R / \gamma$ and the Rayleigh time $t_{R}=\sqrt{\rho R^{3} / \gamma}$, which is the characteristic time scale of an inviscid jet. Others are the intrinsic Deborah number that represents the ratio of the characteristic relaxation time $\lambda$ of the fluid to the Rayleigh time

$$
D e=\sqrt{\frac{\lambda^{2} \gamma}{\rho R^{3}}}
$$

and the elasto-capillary number, $E c=G R / \gamma$, which is the ratio between the elastic modulus of the solution and the capillary pressure. With the general relation between modulus, relaxation time, and polymeric viscosity $\eta=G \lambda$, it is easily shown that for polymeric fluids three dimensionless groups are interrelated via $O h=E c D e$. It should be noted that the dimensionless groups can be determined as a global or a local number by using either the initial or the local value of the radius. The global number is often used to make an initial estimate of the overall thinning dynamics, whereas the local number can be used to predict at which radius a transition from one thinning regime to another is to be expected. Another important dimensionless group compares the relevant thinning velocity to the jet velocity $v_{0}$ to indicate the transition between dripping and jetting dispensing [8]. For low viscous fluids, the Weber number is defined as the squared ratio of the convective time $R_{0} / v_{0}$ to the Rayleigh time

$$
W e=\frac{\rho v_{0}^{2} R_{0}}{\gamma} .
$$

The weakly viscoelastic jets, which are the subject of this paper, exhibit global $D e \approx 1$ and $O h \ll 1$, so viscous stresses can usually be neglected in the following analysis. The temporal evolution of a single instability of the jet is shown in Fig. 1(c), where a characteristic picture is included for each of the four regimes that govern the thinning of the jet. After exiting the nozzle, the jet initially remains almost cylindrical and instabilities slowly arise at the wavenumber associated with the highest growth as predicted by linear stability analysis. The amplitude of this instability grows exponentially and the fluid deformation becomes eventually too large for the linear analysis to remain valid. However, nonlinear similarity solutions to simplified momentum equations can predict the subsequent thinning of the filament that is formed between the two beads. In the second regime, the capillary pressure is dominantly resisted by the inertia of the accelerating fluid elements, resulting in an evolution of the minimal radius $R_{m}$ when approaching the breakup time $t_{p}$ that is described by the inviscid similarity solution [16-18]:

$$
R_{m}=0.64\left(\frac{\gamma}{\rho}\right)^{1 / 3}\left(t_{p}-t\right)^{2 / 3}
$$

The rapid decrease of the filament radius locally induces large strain rates that stretch the polymer molecules in the thread. As a result, the elastic stresses eventually grow large enough to dominate in the third regime the thinning dynamics, and an exponential thinning of the jet in time is observed. This elasto-capillary (EC) regime continues until the polymer chains reach in a fourth regime their finite extensibility limit and the elastic forces cease to grow, which results in a fast breakup [19].

Monitoring this third (exponential) thinning regime of a liquid filament offers a convenient way for measuring submicrosecond relaxation times in extension. The most popular device to measure these extensional properties in polymer solutions is currently the Capillary Breakup Extensional Rheometer (CaBER, Thermo Scientific) that monitors the thinning of a liquid filament that connects two static circular endplates. For this configuration, an analysis of this third regime bridge has shown that the filament radius decreases indeed exponentially in time [20-22] as

$$
R_{m}=\left(\frac{G R_{0}^{4}}{2 \gamma}\right)^{1 / 3} e^{-t / 3 \lambda}
$$

with a distinct time scale of three times the extensional relaxation time $\lambda$ of a viscoelastic solution in this EC balance regime, which allowed in the past to conveniently determine $\lambda$ for a broad variety of (polymeric) fluids [23-28]. 
The CaBER's detection limit is, however, only $1 \mathrm{~ms}$ for low viscous fluids [29]. This restriction is induced by the initial stretching step to create the filament that shifts the position of the narrowest part of the filament away from the position of the laser micrometer. Different studies $[14,30-33]$ have worked on improving the detection limit of these capillary thinning experiments to $\lambda=O(100 \mu \mathrm{s})$ by monitoring the breakup with a high-speed camera and by optimizing the initial stretching distance and velocity. Still, considering that even a limited amount of viscoelasticity has a profound impact on, for instance, the drop-on-demand inkjet printing process [10], it is crucial to have reliable methods to push beyond this limit in order to measure faster relaxation times.

Unlike the filament thinning setup, a continuous jet does not require an initial axial deformation and it should, therefore, be a more appropriate experimental configuration for measuring relaxation times in the microsecond range. A jetting rheometer was originally proposed by Schümmer and Tebel [6], who perturbed the jet at set frequencies and captured the thinning behavior with high-speed photography. Although the theory on the nonlinear behavior of viscoelastic jets was in its infancy, the extensional behavior of polymer solutions could be compared by determining the evolution of the apparent extensional viscosity. In the following decades, the characteristic "beads-on-a-string" structure was simulated and the exponential necking of the ligament connecting the beads was linked to material parameters with various nonlinear constitutive models [20,21]. These results were first applied to extract a relaxation time with a free jetting rheometer by Christanti and Walker [34].

Recently, McKinley and co-workers [35,36] introduced the Rayleigh Ohnesorge Jetting Extensional Rheometer (ROJER) as a new technique to specifically probe the extensional behavior of weakly viscoelastic liquids. In this setup, the cylindrical jet is excited by a piezo-actuator to create a controlled disturbance and the thinning dynamics are visualized with stroboscopic imaging. By selecting a wavenumber that is larger than the most unstable one, the formation of secondary beads between the large droplets is suppressed, resulting in a more precise measurement of the filament radius [37]. Relaxation times down to values as small as $60 \mu$ s have been determined with this setup by fitting the $3 \lambda$ timescale of Eq. (5) to the exponential decrease of the radius $[36,38]$.

However, the use of Eq. (5) to extract the relaxation time is questionable in light of the findings of a recent study by Clasen $e t$ al. that focused on the dripping to jetting transition regime at $W e \approx 1$ of weakly elastic solutions of dilute high molecular weight polymers [39]. While for dripping $(W e \ll 1)$ the timescale $3 \lambda$ has been confirmed [18], with increasing velocity and $W e \geq 1$ eventually a jet develops with a beads-on-a-string morphology where the beads are swallowed by a large terminal drop, which periodically grows and pinches off [39]. Under these conditions, where the EC balance is yet to be established, the thread between the beads should theoretically thin at a different rate, $R_{m} \sim \exp (-t / 2 \lambda)$, and experimentally observed radii appear to follow this new scaling [39].
Naturally, the question arises whether this new time scale of $2 \lambda$ describes also other jetting experiments (as the ROJER technique), or if the so far also for jetting experiments employed time scale of $3 \lambda[34,36,37,40]$ remains correct at higher jet velocities $W e \gg 1$. Quantitative experiments of viscoelastic fluids are scarce [2] and the only direct comparison of relaxation times obtained with jetting and regular capillary thinning experiments on the same fluid has recently been reported by Harlen and co-workers [41]. A reason for this scarcity of experimental data is that the two experiments are probing very different time scales. Despite the fact that jetting rheometry is a suitable measurement technique for weakly viscoelastic liquids, for increasing elasticity the jet quickly becomes too long for resolving the filament radius over the complete exponential thinning regime. This problem does not arise for static thinning filaments in CaBER type devices that are thus suitable for the detection of longer relaxation times. On the other hand, CaBER devices reach their limits for the investigation of short relaxation times due to a fast filament breakup, that is, however, easily captured in a jetting experiment.

A direct and quantitative comparison of the ROJER and CaBER technique to probe the applicable relaxation time scaling laws (which is the aim of this paper) requires the selection of model fluids that exhibit relaxation times that are located near the lower detection boundary of the capillary thinning device. Such a set of model polymer solutions is introduced in Sec. II as well as the employed experimental techniques. The experimental observations of the static capillary thinning and jetting experiments are presented in Sec. III and discussed in Sec. IV, utilizing a numerical and analytical analysis of the filament thinning to demonstrate that there is indeed a difference in the thinning rates in the exponential thinning regime that needs be taken into account in order to extract the correct relaxation time from a ROJER experiment.

\section{MATERIALS AND METHODS}

\section{A. Samples}

The model fluids consist of a series of dilute solutions of PEO (Sigma Aldrich, Bornem, Belgium) with a weight average molecular weight $M_{w}$ of $10^{6} \mathrm{~g} / \mathrm{mol}$ in water. A first set of solutions with three different weight concentrations $c$ of $0.05,0.075$, and $0.1 \mathrm{wt}$. \% are prepared by allowing the polymer to dissolve in distilled water on a rolling bench for $24 \mathrm{~h}$. The samples are shielded from light and all experiments are performed within $72 \mathrm{~h}$ after preparation to minimize degradation of the polymer molecules. The relevant physical and rheological parameters of this first set of model fluids at a temperature of $22^{\circ} \mathrm{C}$ are summarized in Table I. The static interfacial tension $\gamma$ is measured using a $\mathrm{Pt} / \mathrm{Ir}$ Wilhelmy plate connected to an electrobalance (KSV Instruments, Helsinki, Finland). The addition of PEO causes a decrease of the surface tension to a value of $62.4 \mathrm{mN} / \mathrm{m}$, which is independent of the polymer concentration. The shear viscosity $\eta$ is measured with an Ubbelohde viscometer with a capillary with a diameter of $0.53 \mathrm{~mm}$ (Schott Instruments) and the same capillary was used to determine the intrinsic viscosity $[\eta]$ of the polymer. The value of $[\eta]$ 
TABLE I. Physical and rheological properties of the model fluids from the CaBER experiments at a temperature of $22^{\circ} \mathrm{C}$. The relaxation time $\lambda$ is obtained from fitting Eq. (5) to the experimental data of Fig. 3.

\begin{tabular}{|c|c|c|c|c|c|c|c|c|c|c|}
\hline$c($ wt. \%) & {$[\eta](\mathrm{ml} / \mathrm{g})$} & $c / c^{*}$ & $\eta(\mathrm{mPa} \mathrm{s})$ & $\rho\left(\mathrm{kg} / \mathrm{m}^{3}\right)$ & $\gamma(\mathrm{mN} / \mathrm{m})$ & $G(\mathrm{~Pa})$ & $\lambda(\mathrm{ms})$ & $O h$ & $D e$ & $L$ \\
\hline 0.05 & 621 & 0.40 & 1.31 & 998 & 62.4 & 1.08 & $0.97 \pm 0.03$ & 0.0052 & 0.24 & 121 \\
\hline 0.075 & 621 & 0.60 & 1.51 & 998 & 62.4 & 1.62 & $1.36 \pm 0.05$ & 0.0061 & 0.34 & 121 \\
\hline 0.1 & 621 & 0.80 & 1.72 & 998 & 62.4 & 2.16 & $1.78 \pm 0.11$ & 0.0069 & 0.45 & 121 \\
\hline
\end{tabular}

was found by extrapolating a linear fit of six data points of a concentration series of aqueous PEO solutions with relative viscosities $\eta_{r}$ between 1.25 and 2.5 . The intrinsic viscosity is used to evaluate the critical overlap concentration $c^{*}$ of the polymer coils with the expression provided by Graessley [42]: $c^{*}=0.77 /[\eta]$. The $c^{*}$ values in Table I are all in the dilute regime, so that their viscoelastic properties are dominated by the hydrodynamic interactions of the isolated polymer coils and the solvent. For dilute solutions, the elastic modulus $G$ is only a function of the number density of the polymer chains in solution $n=c N_{A} / M_{w}$ :

$$
G=n k_{B} T=\frac{c N_{A} k_{B} T}{M_{w}},
$$

where $N_{A}$ is the Avogadro number, $k_{B}$ is the Boltzmann constant, and $T$ is the absolute temperature [43]. The finite extensibility parameter $L$, representing the ratio of a fully extended polymer coil to its equilibrium length, can be described in terms of molecular parameters as [30]:

$$
L=\sqrt{3}\left(\frac{j \sin ^{2}\left(\theta_{b} / 2\right) M_{w}}{C_{\infty} M_{u}}\right)^{1-v},
$$

where $\theta_{b}$ is the average bond angle in the monomer, $j$ is the number of bonds of a monomer of molar mass $M_{u}$, and $C_{\infty}$ is the characteristic ratio for a given polymer-solvent system. The values for PEO are $\theta_{b}=109.4^{\circ}$ (for which the C-C bond angle was taken), $j=3, M_{u}=44 \mathrm{~g} / \mathrm{mol}$, and $C_{\infty}=4.1$ [44].

To extend the results obtained with the model fluids to different molecular weights and solvent viscosities, a second series of solutions is used to quantitatively compare CaBER and jetting experiments in which the jet is excited by a piezo-actuator for the higher viscous solutions. The samples consist of dilute solutions of PEO with average molecular weights of $3 \times 10^{5}, 6 \times 10^{5}$, and $10^{6} \mathrm{~g} / \mathrm{mol}$ in water-glycerol solutions, with 25 and $40 \mathrm{wt}$. \% glycerol contents, respectively. According to the evaluated $c^{*}$, all solutions are in the diluted regime and the reduced concentrations vary between 0.007 and 0.33. Shear viscosities are determined with a stress-controlled rheometer (AR-G2, TA Instruments) and a double-wall Couette geometry. The static surface tension is measured using the pendant drop method (CAM 200, KSV Instruments). The relevant physical properties of these solutions are summarized in Table IV.

\section{B. Capillary breakup experiments}

The necking of a single liquid bridge is monitored with the CaBER-1 (Thermo Haake GmbH, Karlsruhe, Germany). The experiments are executed at an ambient temperature of
$22{ }^{\circ} \mathrm{C}$ with circular disks of radius $R_{0}=2 \mathrm{~mm}$ and with an initial gap distance of $L_{0}=2 \mathrm{~mm}$. These small disks are chosen to reduce gravitational sagging. Gravitational effects lead to a weak axial flow, such that more than half of the initial sample volume is found below the mid-plane. Since the analysis of the filament thinning always presumes an axial symmetry, this drainage should be minimized. For the CaBER experiment, the Eötvös or Bond number $E o=B o=\rho g R_{0} L_{0} / \gamma$, which expresses the ratio of gravitational to capillary forces, has an initial value of $B o \approx 1$ for the given dimensions. During the thinning process, the filament radius and thus the local Bond number will decrease, so the filament is eventually only drained by capillary forces [45]. The fluid samples are carefully loaded between the plates with a syringe to avoid air bubbles in the fluid.

The unstable liquid bridge is created by stretching in a linear motion over a timespan of $50 \mathrm{~ms}$. The initial gap is kept fixed at $L_{0}=R_{0}=2 \mathrm{~mm}$, and the final gap height equals $L_{1}=5 \mathrm{~mm}$, which is only slightly above the upper stability limit $L_{1}=2.25 R_{0}$ of a liquid cylinder [46]. This small stretch step creates very short filaments with an inherently high axial curvature, so that initially the self-similar scaling laws do not apply, as they are based on a slenderness approximation [30]. As the filament gets thinner, it evolves to a slender shape and the extracted radii can eventually be used to determine the extensional flow properties from the appropriate similarity solution.

The evolution of the minimal filament radius $R_{m}$ is not monitored with the laser micrometer of the CaBER. The laser micrometer only captures the mid-point radius, which is not necessarily the minimal radius for low viscous, complex fluid threads [29]. Instead, the thinning dynamics are determined with a high-speed camera (Fastcam SA2, Photron, USA) to better capture the heterogeneous nature of the filament near breakup. The camera is connected to a tube lens system equipped with $5 \times$ microscopic objectives (Olympus, Japan) to obtain high resolution images $(1.9 \mu \mathrm{m} / \mathrm{pixel})$. Illumination is provided with a fiber-optic illuminator Fiber-Lite DC-950 (Dolan-Jenner Industries, Boxborough, MA, USA) and a 50 $\mathrm{mm}$ condenser lens. Images are taken at a rate of $3000 \mathrm{fps}$ with a shutter time of $10 \mu \mathrm{s}$ and are analyzed with the self-written, MATLAB-based image processing algorithm EdgeHog [31].

\section{Jetting experiments}

Figure 2 shows the experimental setup used to study the jet breakup of a polymer solution. A syringe containing $50 \mathrm{ml}$ of the sample fluids is placed in a syringe pump (Harvard Apparatus, Holliston, MA, USA) to deliver fluid at a constant flow rate and is connected with a flexible tube to a nozzle 


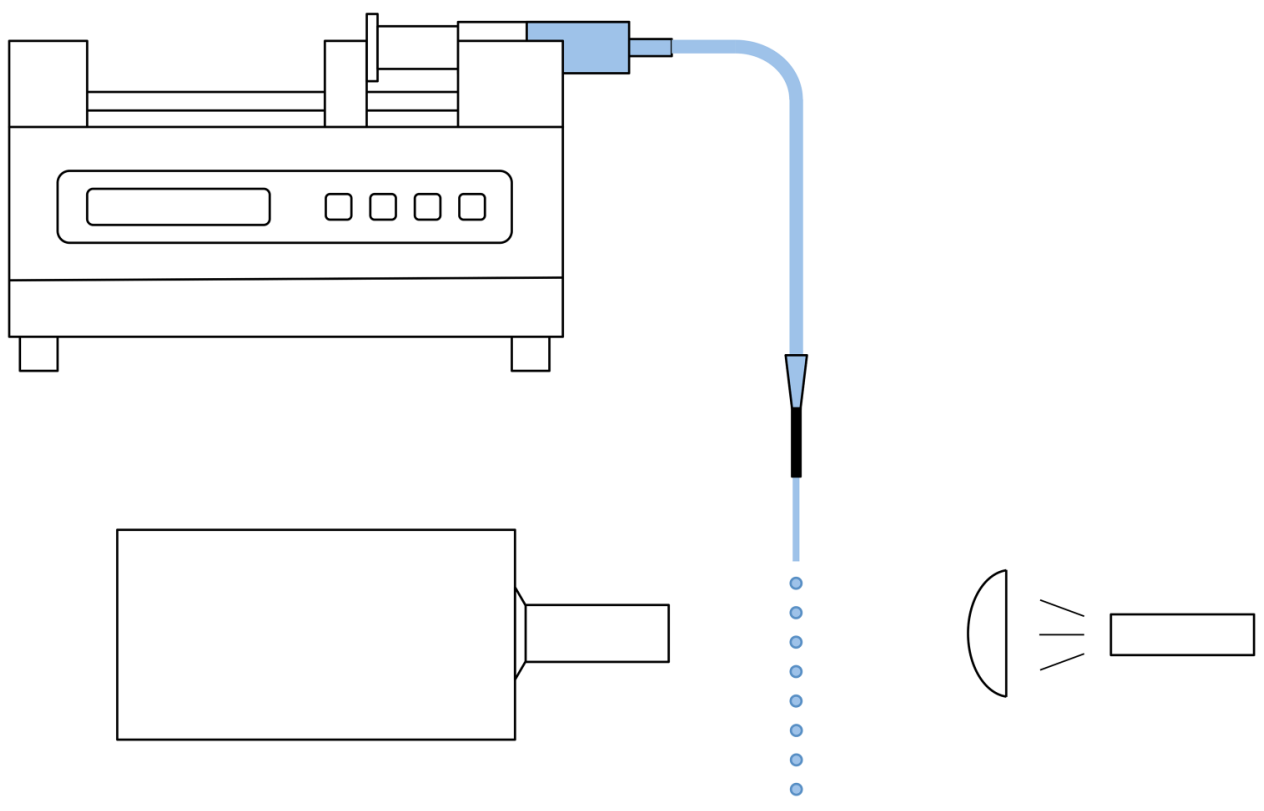

FIG. 2. Schematic image of the experimental setup for the jetting tests.

(Nordson EFD precision tips). In a first set of jetting experiments with the model fluids, two different nozzles with diameters of 0.15 and $0.25 \mathrm{~mm}$ are used throughout the experiments and the most unstable perturbation is allowed to grow naturally on the jet surface. For the second set of experiments with higher viscous water-glycerol solutions, a $0.20 \mathrm{~mm}$ nozzle clamped against a piezoelectric stack actuator (Pst 150, APC International) is used in order to force a controlled disturbance on the jet. Frequency, shape, and amplitude of the periodic perturbation are specified through a function generator (1250 Frequency Response Analyser, Schlumberger) and a voltage amplifier (S-100 MK II, Thomann). The thinning dynamics of the jet are visualized with a high-speed camera (Fastcam SA-2, Photron, San Diego, CA, USA) connected to one of two employed lens systems. The first lens system, a tube lens/microscopic objective assembly, is the same as employed for the CaBER in order to directly compare variations in the filament shape evolution between the two experiments. Consequently, the field of view of this lens is limited to $1.46 \times 2.37 \mathrm{~mm}$ and the jet was captured with multiple images. For this, the nozzle is mounted on a twodimensional linear stage driven by a micrometer screw, allowing a precise displacement of $50 \mathrm{~mm}$ in the vertical direction. The second lens system is a lower magnification $55 \mathrm{~mm}$ focal length telecentric lens (TEC-M55 Computar, CBC, Tokyo, Japan) with a spatial resolution of $9.5 \mu \mathrm{m} / \mathrm{pixel}$ and a maximum field of view of $15 \times 15 \mathrm{~mm}^{2}$ and is used to capture the breakup mechanism of jets with higher velocities.

All experiments are executed according to the same protocol: first a stable flow is ensured by waiting $90 \mathrm{~s}$ and the jet is subsequently captured by taking a series of pictures of its different sections. The images are taken at rates ranging from 3000 to 3600 frames/s with a shutter time of $2.7 \mu \mathrm{s}$. A typical image of a jet thinning without application of an external frequency and thus asperities developing at the natural frequency is shown in Fig. 1(a). A slight nonequidistance between the beads arises from ambient nose. Figure 1(b) depicts then a jet excited with its natural frequency and equidistant beads.

The image processing is carried out with a self-written MATLAB-based algorithm. The edges of the jet are detected with a robust Canny edge detector [47] and the local jet radius is used to determine the position of the instabilities in each frame. For each instability, the minimal radius $R_{m}$ is determined with sub-pixel accuracy using a modified Marr-Hildreth algorithm [31,48]. Because the jet velocity can be determined from the flow rate and the initial jet radius, the minimal radii of the same instability can be tracked over the subsequent images, so that the minimum radius is directly linked to a location on the jet, usually expressed as a distance from the nozzle. The temporal evolution of each single instability is thus separately obtained. The presented data extracted from each single instability evolution is then averaged over at least 10 single experiments for each flow rate. It should be noted that this procedure is different from the ROJER experiments performed by McKinley and co-workers [36,40], where they used a stroboscopic technique with a slight difference between a multiple of the strobing frequency and the excitation frequency of the jet to observe the instabilities and their $R_{m}$ at slightly varying positions (distances from the needle). Their approach is, however, not following the same instability along the jet as the strobing frequency is much lower than the excitation frequency. Our approach, on the other hand, allows to observe also an unexcited jet, as slight variations in the natural frequency of instability development, as observed, for example, in Fig. 6(a), do not matter when tracing a single instability over multiple images.

\section{EXPERIMENTAL RESULTS}

\section{A. Capillary breakup experiments}

The extensional flow properties of the model fluids are first characterized with CaBER experiments. The large spatial and temporal resolution of the setup allows an 
accurate determination of the characteristic relaxation time of the fluids, which is used in Sec. III B to evaluate the time scales during the jet breakup. Figure 3 shows the evolution of the minimal radius of the filament during the experiment for the three solutions of PEO. The initial type of decrease of the filament radius is predicted by the global Ohnesorge numbers in Table I, which are calculated using the estimated radius at the cessation of stretching that was determined by a lubrication solution for a Newtonian fluid

$$
R_{m}=R_{0}\left(\frac{L_{1}}{L_{0}}\right)^{-3 / 4}
$$

with $L_{0}$ and $L_{1}$ being the initial and the final gap, respectively [49]. By comparing the characteristic thinning velocities of the viscosity and inertia dominated regimes, the boundary between the two regimes is located at a critical value $O h^{*}=$ 0.2077 [30]. The values for the model solutions in Table I are far below this critical value, so the capillary pressure is initially only resisted by fluid inertia. The fluid continues to accelerate and appears to approach a finite-time singularity at the pinching time $t_{p}$, as described by Eq. (4) and represented by the dashed line in Fig. 3. The increasing strain rate in the fluid neck is eventually high enough to stretch the polymers and to generate a buildup of elastic stresses that start to dominate the fluid inertia and suppress the singularity. This transition marks the onset of the EC regime where the filament radius decreases exponentially in time following Eq. (5), resulting in a constant strain rate, which is inversely proportional to the longest relaxation time $\lambda$. In order to better distinguish the differences in the EC regime, the curves in Fig. 3 are shifted by $t_{p}$ along the time axis, so that the initial inertiacapillary regimes collapse for the three fluids. For lower polymer concentrations, the transition occurs at smaller radii, suggesting that a higher strain is necessary to reach sufficiently high elastic stresses. The characteristic relaxation time $\lambda$ of each solution is extracted from the thinning rate in the EC regime using the $3 \lambda$ time scale of Eq. (5). The relaxation time increases with polymer concentration and the values are listed with the standard deviation (obtained from at least 10 different experiments for each concentration) in Table I.

The onset of the EC regime can also be seen in the shape of the filament in Fig. 4. The asymmetric shape that characterizes inertia resisted thinning disappears at the start of the EC regime and a cylindrically shaped filament emerges. The shape remains axially uniform for the two highest concentrations, whereas the $0.05 \mathrm{wt} \%$ solution shows significant necking at both the top and the bottom droplet, generating a large bead in the middle of the filament. Criteria for the occurrence of a central satellite bead in viscoelastic filaments were introduced by Wagner et al. [50], based on the asymmetry induced by the self-similarity of the pinching process. Small differences in radius and hence in capillary pressure between the short filaments above and below this central bead impel the bead to coalesce with one of the two fluid reservoirs. The formation of a central bead was also observed by Tirtaatmadja et al. [18] during dripping experiments on a series of dilute solutions of PEO with varying molecular weight in glycerol-water mixtures. The bead disappeared for



FIG. 3. Temporal evolution of the minimal filament radius during capillary breakup experiments of three polymer solutions. The dashed line indicates a power law fit of the inertia-capillary regime [Eq. (4)] and the straight lines represent an exponential fit used to extract the dominant relaxation time $\lambda$ [Eq. (5)].

the higher molecular weight samples, which exhibited higher relaxation times $\lambda$ and thus higher Deborah numbers. Bhat et al. [15] found a comparable trend in simulations of the breakup of viscoelastic filaments. Satellite beads only appeared in the case of sufficient inertia $(O h<1)$ and moderate elasticity $(D e<0.3)$. In fluids with a comparable Ohnesorge number as our model fluids, bead formation is more pronounced for lower Deborah numbers, which agrees with our observations. Moreover, the presence of the bead does not appear to affect the thinning dynamics, as no deviations from the exponential decay are observed in Fig. 3. The minimal radius can be tracked for all solutions until a value of $5 \mu \mathrm{m}$. At this point the filament morphology changes dramatically, forming a characteristic blistering structure as described by Oliveira et al. [51] and Sattler et al. [52], caused by a relaxation of the fully stressed state into a partially relaxed state of the filament before pinching off.

\section{B. Jetting experiments}

Following the study of the CaBER breakup behavior of the three polymer solutions and the characterization of the relaxation times in the EC balance, jetting flows of the model fluids are examined in three sets of experiments. In a first set, the three PEO solutions are emitted at approximately the same jet velocity $(W e \approx 8)$. Despite the fact that the same flow rate is chosen for all solutions, small differences in the initial radius $R_{0}$ of the jet after exiting the nozzle cause slight variations in the actual jet velocity. A higher polymer concentration increases the initial jet radius and hence lowers the actual jet velocity expressed by the Weber number (see Table II). As shown in Fig. 5, all fluids exhibit a similar thinning behavior as in the regular CaBER capillary thinning experiments. Only the initial radius of the filament is notably smaller, because the jet originates from a nozzle with an inner radius $R_{n}=75 \mu \mathrm{m}$. Similar to the CaBER experiments, the jetting tests exhibit an exponential thinning of the radius at intermediate times:

$$
R_{m} \sim e^{-t / \theta} .
$$


(a)
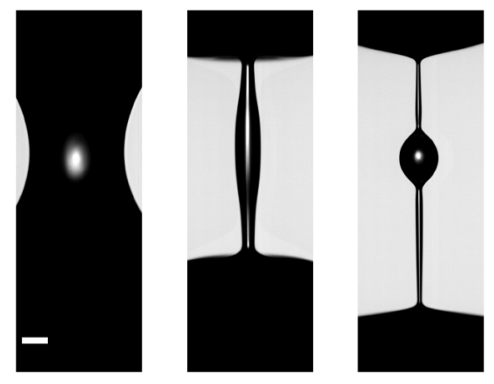

(b)
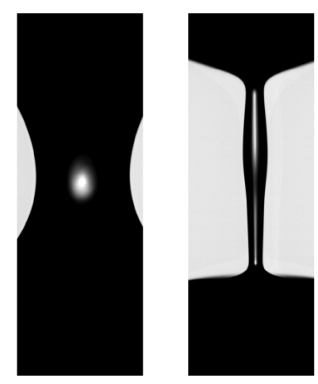

(c)
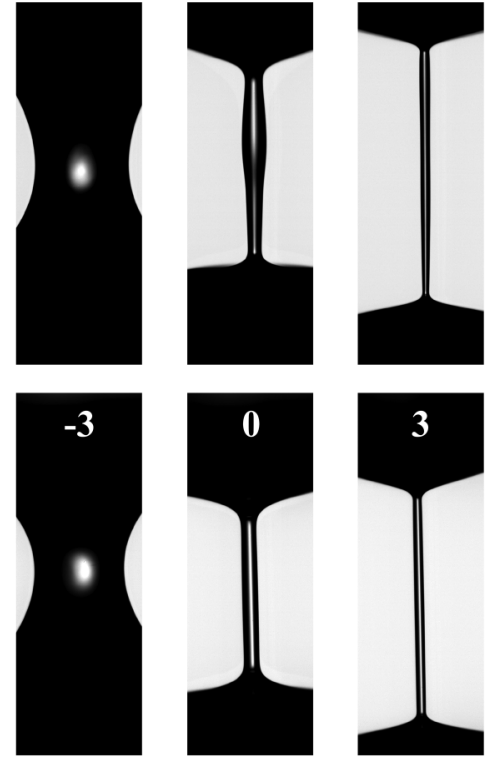
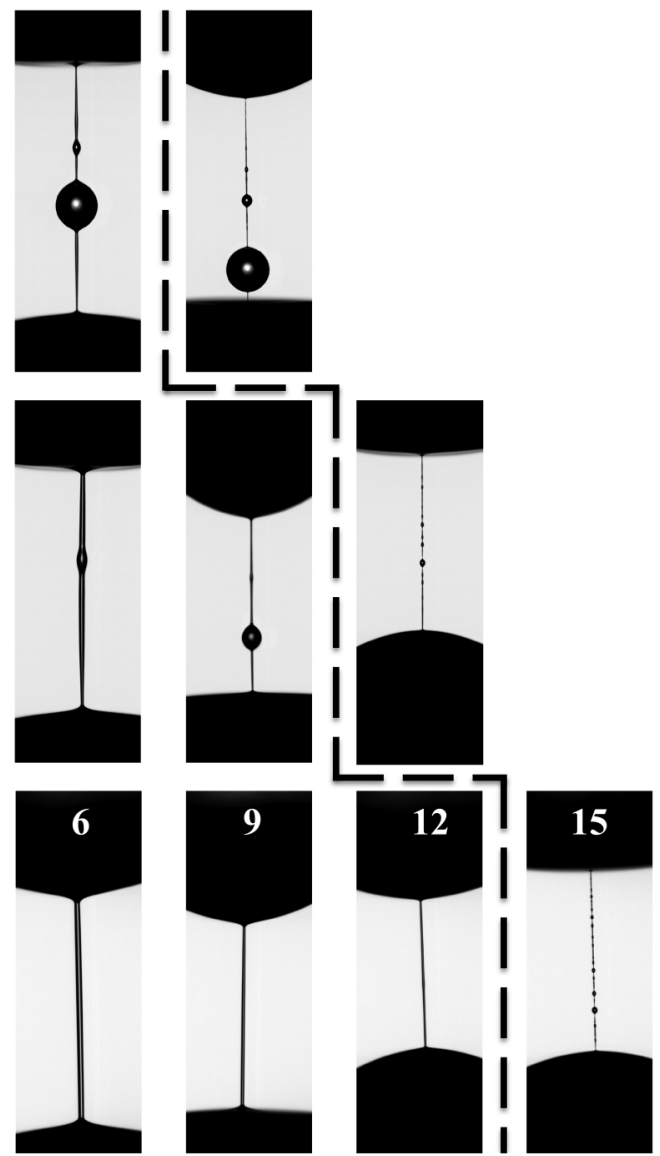

FIG. 4. Capillary thinning and breakup of a filament in a CaBER setup for aqueous PEO solutions of (a) 0.05 wt. \%, (b) 0.075 wt. \%, and (c) 0.1 wt. $\%$. The shifted time $t-t_{p}(\mathrm{~ms})$ is displayed and the scale bar represents a distance of $200 \mu \mathrm{m}$. The dashed line indicates the resolution limit of the lens.

The more concentrated solutions thin at a lower rate, revealing an increase of the time scale $\theta$ in the exponential thinning regime. However, it is striking that the minimal jet radius $R_{m}$ decreases faster for the same sample in comparison to the CaBER experiments. The different thinning rate in the exponential regime of both experiments is emphasized in the inset of Fig. 5 where the time is rescaled with the relaxation time $\lambda$ that was obtained during the CaBER experiments, and the radius with $R_{E C}$ at the onset of an exponential thinning. Using these scalings the filament radii of the three solutions measured using the CaBER devise exhibit the same slope of $-1 / 3 \log (e)$ in the inset.

In contrast, the jetting experiments display a steeper slope, which is approximately the same for all samples. This observation demonstrates that the time scale $\theta$ in the exponential regime of the jetting experiments is proportional to the relaxation time $\lambda$. To accurately determine this scale, at least ten instabilities are followed in time for each sample to obtain an average value of $\theta$, which is listed with the standard

TABLE II. Overview of the parameters of the jetting experiments with the three PEO solutions.

\begin{tabular}{lccccc}
\hline \hline$c($ wt. \%) & $\dot{Q}(\mathrm{ml} / \mathrm{min})$ & $R_{0}(\mu \mathrm{m})$ & $W e$ & $\theta(\mathrm{ms})$ & $\theta / \lambda$ \\
\hline 0.05 & 3.17 & 80 & 8.9 & $2.0 \pm 0.1$ & $2.1 \pm 0.1$ \\
0.075 & 3.17 & 82 & 8.3 & $2.7 \pm 0.1$ & $2.0 \pm 0.1$ \\
0.10 & 3.17 & 85 & 7.2 & $3.7 \pm 0.1$ & $2.1 \pm 0.2$ \\
\hline
\end{tabular}

deviation in Table II. The time scale is determined with a reduced accuracy of only two significant digits because the fits of the exponential decay are less precise than for the CaBER experiments. This lower precision is caused by the lower resolution of the telecentric lens that is used for the jetting experiments. The ratio of the time scale with the relaxation time $\theta / \lambda$ is determined for each sample and an approximation of the standard deviation of the ratio of two normally distributed variables is included in Table II $[53,54]$. A time scale $\theta \approx 2 \lambda$ is observed during the jetting of all samples. As a result, the reference slope of $-1 / 2 \log (e)$ agrees quantitatively with the decay measured using the ROJER.

Further jetting experiments are conducted to investigate the effect of the Weber number. The $0.1 \mathrm{wt}$ \% PEO solution is examined for a range of Weber numbers, and the evolution of the minimal filament radius $R_{m}$ is shown as a function of the distance from the needle in Fig. 6(b). The lowest flow rate $(W e=1.3)$ was chosen close to the dripping-jetting transition and some of the beads still appear to coalesce just before breakup at this flow rate [see Fig. 6(a)]. This experiment is thus performed at the boundary of the gobbling regime for which a thinning with a time scale of $2 \lambda$ was initially proposed [39].

The evolution of the minimal radius $R_{m}$ is followed during the exponential thinning regime for all flow rates using the optical setup that is able to capture a section of the jet with a length of approximately $15 \mathrm{~m}$. However, for small Weber numbers this field of view is sufficient to monitor the 


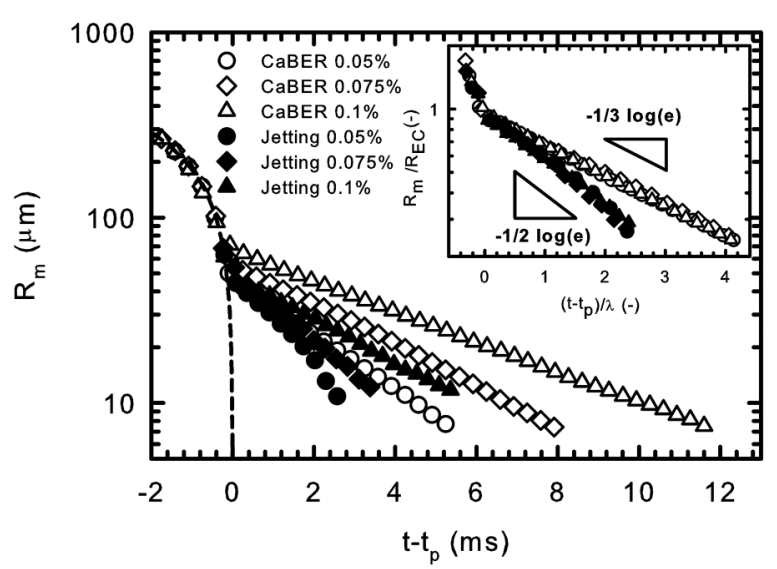

FIG. 5. Development of the minimal filament radius during the exponential thinning regime in a regular CaBER capillary thinning experiments, and in jetting experiments at $W e \approx 8$ and from a nozzle of $0.15 \mathrm{~mm}$ diameter for three PEO solutions. The evolution of the radius is presented in the same way as in Fig. 3. In the inserted graph, the radius is rescaled with the radius $R_{E C}$ at the onset of an exponential thinning, and the time is rescaled with the relaxation time $\lambda$ obtained with the CaBER, to emphasize the different time scales in both experiments.

complete nonlinear thinning dynamics of the jet, including the inertia resisted regime. A single frame can capture a segment of the jet from the origin of the capillary instability until the late stages of the exponential thinning regime. As the Weber number increases, the initial straight segment lengthens, which is shown in the images as well as in the position of the onset of the exponential thinning in Fig. 6(b). Additionally, the length of the exponential thinning regime increases due to the higher jet velocity and the jet becomes too long to track the complete thinning of an instability for intermediate flow rates $(W e>5)$.

Further increasing the flow rate results in the acquisition of even fewer data points and eventually the exponential regime can only be partially captured in a single image for high flow rates $(W e>15)$. Since the diameter of larger filaments can be determined more accurately, we focus on the beginning of the exponential thinning regime for these flow rates. Jetting experiments could be carried out until the Weber number reaches a value of 70 . At this highest jet velocity, the instability can only be followed for seven consecutive frames, which is the lower boundary for reliably fitting the exponential thinning regime (Table III).

The same data set of jetting experiments is presented in Fig. 7 as a function of time after exiting the nozzle, rescaled with the relaxation time $\lambda$ from the CaBER measurements. All jetting experiments exhibit the same thinning behavior in the exponential regime. The average slope of the decreasing radii in the exponential thinning regime agrees with the reference slope of $-1 / 2 \log (e)$ for all We (see Table III). Note that only part of the exponential thinning regime can be observed for the two highest flow rates, yielding a larger standard deviation.

We now investigate the effect of nozzle radius. The initial jet radius $R_{0}$ is larger than the nozzle radius for all our jetting experiments due to the extrudate swell effect. The swell is more pronounced for more concentrated samples, since these have longer relaxation times and thus display stronger elastic
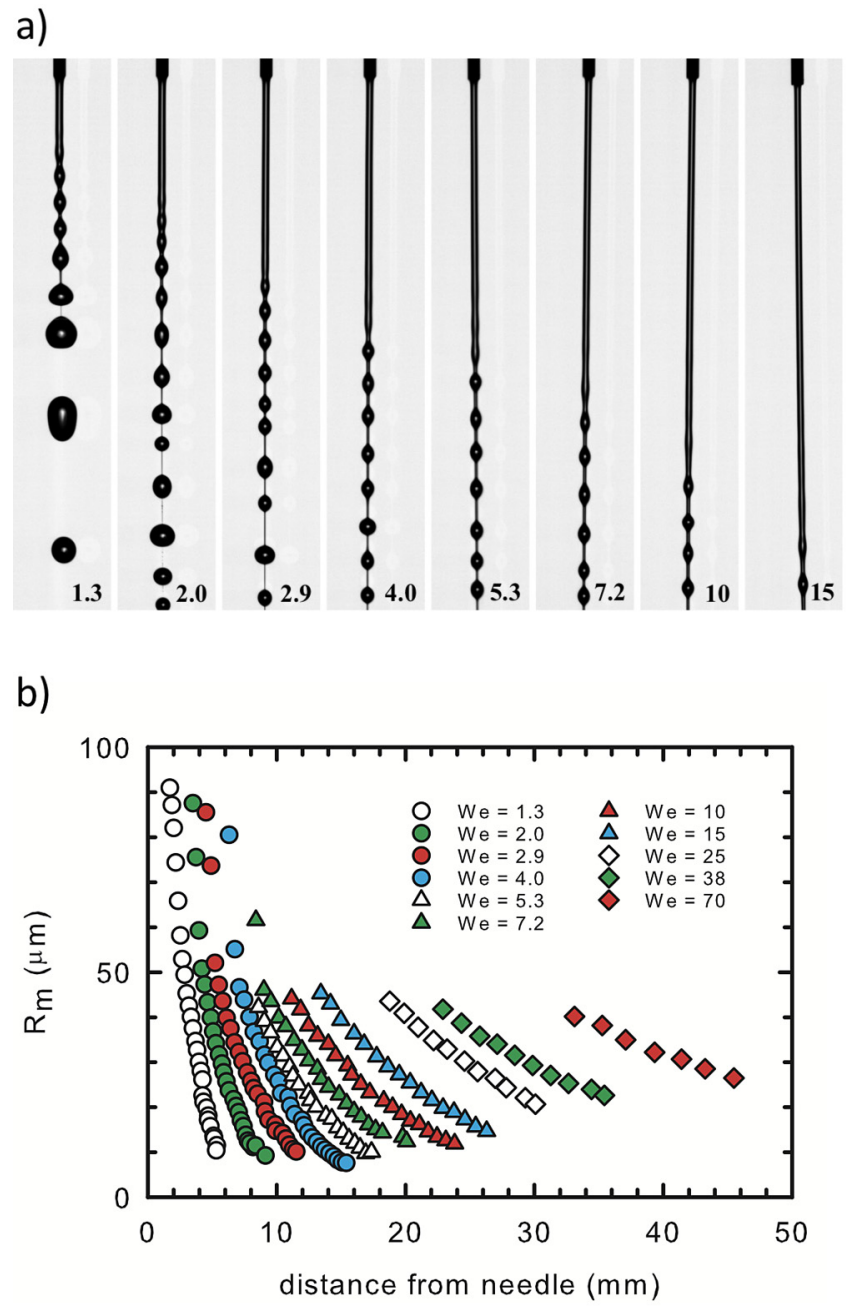

FIG. 6. Thinning dynamics of the 0.1 wt. $\%$ PEO solution jetted from a nozzle of $0.15 \mathrm{~mm}$ diameter for different Weber numbers. (a) Images of the different jets with the Weber number in the bottom corner. (b) Development of $R_{m}$ of single filament instabilities for different Weber numbers, each followed over the whole field of view of $15 \mathrm{~mm}$ and represented as a function of the distance from the needle. For this, the imaging system was moved along the jet to focus on the exponential thinning regime before filament breakup.

behavior. The swelling further depends on the needle size because the shear rate near the needle wall rapidly increases as the needle radius decreases. Figure 8 compares the evolution of the minimal filament radius $R_{m}$ for jets of the same 0.1 wt. \% PEO solution exiting from two different needles. The initial jet radius $R_{0}$ is clearly different for both experiments and appears to scale with the nozzle radius $R_{n}$. Additionally, the larger nozzle causes the IC regime as well as the exponential thinning to start at a larger radius. The time scale $\theta$ of the exponential decrease of the jet radius is, however, identical for both nozzle sizes with $\theta \approx 2 \lambda$.

Lastly, the observation of the thinning timescale $\theta \approx 2 \lambda$ for jets is verified for a range of polymer molecular weights, concentrations, and solvent viscosities. These jetting experiments are performed on solutions of PEO with $M_{w}=$ $3 \times 10^{5}$ to $1 \times 10^{6} \mathrm{~g} / \mathrm{mol}$ in water-glycerol mixtures of different compositions and concentration from 0.001 to 0.1 wt. \% (exact compositions and resulting viscosities are given in Table IV), while applying a periodic disturbance to 
TABLE III. Overview of the jetting experiments with the $0.1 \mathrm{wt} \%$ aqueous PEO solution from a nozzle of $0.15 \mathrm{~mm}$ diameter. The jet velocity is varied from a value near the dripping-jetting transition $(W e=1.3)$ to the experimental limit of the setup $(W e=70)$.

\begin{tabular}{lcccc}
\hline \hline $\begin{array}{l}\dot{Q} \\
(\mathrm{ml} / \mathrm{min})\end{array}$ & $\begin{array}{c}R_{0} \\
(\mu \mathrm{m})\end{array}$ & $W e$ & $\begin{array}{c}\theta \\
(\mathrm{ms})\end{array}$ & $\theta / \lambda$ \\
\hline 1.60 & 95 & 1.3 & $1.8 \pm 0.1$ & $2.0 \pm 0.2$ \\
1.90 & 93 & 2.0 & $1.8 \pm 0.1$ & $2.0 \pm 0.2$ \\
2.22 & 91 & 2.9 & $1.9 \pm 0.2$ & $2.2 \pm 0.2$ \\
2.54 & 89 & 4.0 & $1.8 \pm 0.1$ & $2.1 \pm 0.2$ \\
2.85 & 88 & 5.3 & $1.8 \pm 0.1$ & $2.1 \pm 0.2$ \\
3.17 & 85 & 7.2 & $1.8 \pm 0.1$ & $2.1 \pm 0.2$ \\
3.70 & 83 & 10 & $1.9 \pm 0.1$ & $2.1 \pm 0.2$ \\
4.23 & 81 & 15 & $1.8 \pm 0.1$ & $2.1 \pm 0.2$ \\
5.29 & 79 & 25 & $1.9 \pm 0.1$ & $2.1 \pm 0.2$ \\
6.34 & 78 & 38 & $2.0 \pm 0.2$ & $2.3 \pm 0.3$ \\
8.46 & 77 & 70 & $2.0 \pm 0.2$ & $2.2 \pm 0.3$ \\
\hline \hline
\end{tabular}

be able to measure the higher viscosity solutions. The imposed frequency is always chosen approximately 100$200 \mathrm{~Hz}$ higher than the frequency corresponding to the most unstable wavelength, calculated from the dispersion relation for viscoelastic jets derived by [4]. A typical image of such a jet is given in Fig. 1(b). The relaxation times are calculated from the jetting experiments using the new time scale in Eq. (9) of $\theta=2 \lambda$. The results are presented in Fig. 9, scaled for convenience with the Zimm time $\lambda_{\text {Zimm }}=0.463 \frac{\eta_{S}[\eta] M_{w}}{N_{A} k_{b} T}$ to compare different molecular weights and solvent viscosities on the same master curve as function of the reduced concentration, in agreement with the correlation $\frac{\lambda}{\lambda_{\text {zimm }}} \sim\left(\frac{c}{c^{*}}\right)^{m}$ initially proposed by [18]. Figure 9 shows the scaled relaxation times for both jetting and for separately conducted CaBER experiments (using $\theta=3 \lambda$ ) on the same solutions. The experimental data show a very good agreement, evidence that the new time scale for jetting experiments of $\theta=2 \lambda$ is also recovered for different molecular weights up $M_{w}=10^{6} \mathrm{~g} /$ mol and different solvent viscosities, as well as when a controlled disturbance is applied.

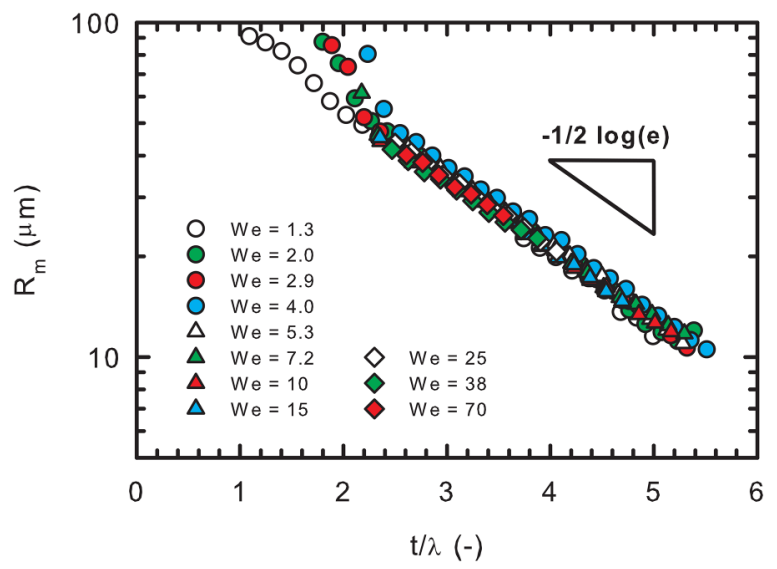

FIG. 7. Thinning dynamics of the 0.1 wt. $\%$ PEO solution for different Weber numbers. The data points of Fig. 6(b) are shown as a function of the ratio of time to the relaxation time $\lambda$ obtained with the CaBER. The radii appear to collapse in the exponential thinning regime for different Weber numbers and the slope in this regime corresponds to the reference slope, representing a thinning with a time scale of $2 \lambda$.
TABLE IV. Composition of the solutions for jetting experiments with different molecular weight of PEO, concentrations, and solvent viscosities used in Fig. 9.

\begin{tabular}{lcccccc}
\hline \hline $\begin{array}{l}M_{w} \\
(\mathrm{~kg} / \mathrm{mol})\end{array}$ & $\begin{array}{c}c \\
(\text { wt. } \%)\end{array}$ & $c / c^{*}$ & $\begin{array}{c}\text { Water/glycerol } \\
\text { ratio }\end{array}$ & $\begin{array}{c}\eta \\
(\mathrm{mPa} \mathrm{s})\end{array}$ & $\begin{array}{c}\rho \\
\left(\mathrm{kg} / \mathrm{m}^{3}\right)\end{array}$ & $\begin{array}{c}\gamma \\
(\mathrm{mN} / \mathrm{m})\end{array}$ \\
\hline 300 & 0.100 & 0.340 & $75 / 25$ & 2.30 & 1059.2 & 62.0 \\
600 & 0.053 & 0.010 & $75 / 25$ & 2.12 & 1059.2 & 62.5 \\
600 & 0.010 & 0.053 & $60 / 40$ & 3.61 & 1098.8 & 63.4 \\
600 & 0.025 & 0.133 & $60 / 40$ & 3.60 & 1098.8 & 63.4 \\
1000 & 0.005 & 0.037 & $75 / 25$ & 1.99 & 1059.2 & 62.5 \\
1000 & 0.001 & 0.007 & $60 / 40$ & 3.57 & 1098.8 & 63.3 \\
1000 & 0.005 & 0.037 & $60 / 40$ & 3.57 & 1098.8 & 63.4 \\
\hline \hline
\end{tabular}

To summarize, we have detected a significant difference between the thinning rates of regular capillary breakup and jetting experiments. Contrary to the generally employed assumption that the final thinning dynamics of these freesurface flows are similar, the experiments presented in this section clearly demonstrate that a weakly viscoelastic jet (as utilized in a ROJER experiment) breaks up faster than a capillary bridge of the same liquid in a CaBER experiment. The difference is mainly situated in the exponential thinning regime, where in the ROJER experiment a different time scale for the exponential decrease of the filament radius is observed:

$$
R_{m} \sim \exp (-t / 2 \lambda)
$$

This new time scale $\theta=2 \lambda$ was already suggested for capillary jets near the dripping-jetting transition $(W e \approx 1)$ [39], but it is here demonstrated that this scaling is valid for low to moderate Weber numbers up to $W e=70$.

\section{DISCUSSION}

To determine the origin of this difference in breakup dynamics, an analytical and numerical analysis of the thinning filament is conducted for both setups. We start our analysis from a simple zero-dimensional force balance that is introduced in Sec. IV A.

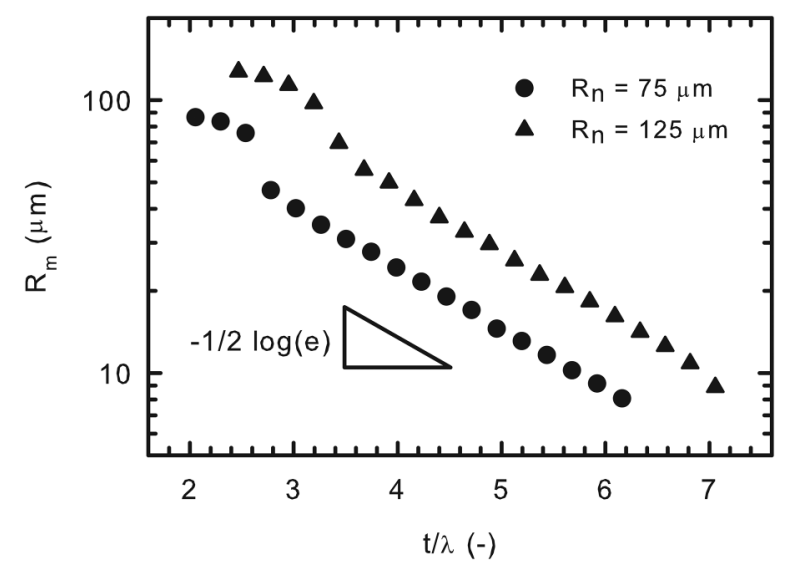

FIG. 8. Comparison of the thinning dynamics of a $0.1 \mathrm{wt} \%$ PEO jet with two different nozzles at $W e \approx 3$. 


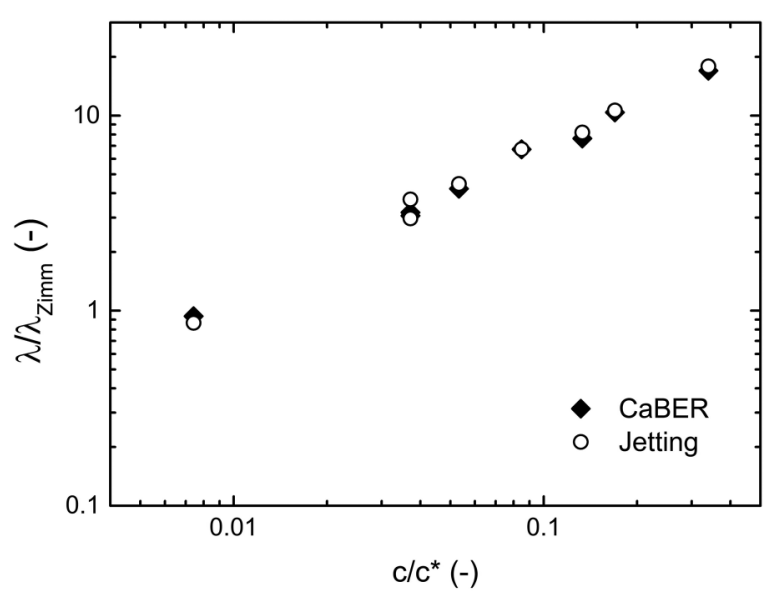

FIG. 9. Scaled relaxation times $\lambda / \lambda_{\text {Zimm }}$ as function of reduced concentration $c / c^{*}$ for different PEO molecular weights and solvent viscosities as detailed in Table IV. For jetting experiments, the Weber numbers vary between 15 and 25 , and the imposed frequency is in the range $3-5 \mathrm{kHz}$. The jetting relaxation times are determined with Eq. (9) and $\theta=2 \lambda$, whereas the $\lambda$ obtained with the CaBER use $\theta=3 \lambda$.

\section{A. Stress balance for a viscoelastic filament}

We start our analysis from the Navier-Stokes equation for an incompressible fluid by assuming that the filament or jet is a slender axisymmetric volume. The flow in the slender filament is then described with the full leading order momentum balance, taking the full description of the curvature into account, as introduced by Eggers and Dupont [55], including an inertial term as well as a polymeric stress contribution [22]:

$$
\begin{aligned}
& \frac{\partial}{\partial \tau}\left(h^{2} \bar{v}\right)+\frac{\partial}{\partial \bar{z}}\left(h^{2} \bar{v}^{2}\right) \\
& \quad=\frac{\partial}{\partial \bar{z}}\left(h^{2}\left(K+3 O h_{s} \frac{\partial \bar{v}}{\partial \bar{z}}+\Delta \bar{\sigma}_{p}+\frac{\dot{h}^{2}}{2}\right)\right)-h^{2} B o \\
& \quad=\frac{1}{\pi} \frac{\partial \bar{F}_{z}}{\partial \bar{z}}
\end{aligned}
$$

To identify the relative importance of the different terms in this balance, the parameters have been nondimensionalized by introducing the dimensionless radius $h=R / R_{0}$ (and the dimensionless derivative thereof $\dot{h}$, utilizing the dimensionless time $\tau=t / t_{R}$, where $t_{R}=\sqrt{\rho R_{0}^{3} / \gamma}$ is the Rayleigh time scale), the dimensionless velocity $\bar{v}=v t_{R} / R_{0}$ and axial coordinate $\bar{z}=z / R_{0}$, the dimensionless axial force $\bar{F}_{z}=F_{z} /\left(\gamma R_{0}\right)$, and the dimensionless polymeric normal stress difference $\Delta \bar{\sigma}_{p}=\Delta \sigma_{p} R_{0} / \gamma, \quad$ where $\Delta \sigma_{p}=\sigma_{p, z z}-\sigma_{p, r r}$. The Ohnesorge number $O h_{s}$ is based on Eq. (1) but uses the solvent viscosity and is related to the other dimensionless groups as

$$
O h_{s}=\frac{E c D e}{\eta_{s p}}
$$

with $\eta_{s p}=\left(\eta-\eta_{s}\right) / \eta_{s} . K$ is the curvature term that is defined as $[22,56,57]$

$$
K=\frac{1}{h \sqrt{1+(\partial h / \partial \bar{z})^{2}}}+\frac{\partial^{2} h / \partial \bar{z}^{2}}{\left(1+(\partial h / \partial \bar{z})^{2}\right)^{3 / 2}} .
$$

Since we focus on the elasticity controlled thinning regime, in which the filament forms a uniform cylindrical column with a constant radius $R$, the mean curvature reduces here to $K=1 / h$. Full curvature can be included in the following analysis and does not change the physical picture. Gravitational acceleration is negligible for the small dimensions of the jet considered here, which can be easily determined from the Bond number $B o=\rho g R_{0}^{2} / \gamma$, so that we drop this term on the right-hand side of Eq. (11) for the following analysis.

The left-hand side of Eq. (11) is the material derivate of a Lagrangian element of the fluid filament, whereas the term in brackets on the right-hand side represents the axial force $F_{z}$ acting on a cross section of the filament. A simple integration, dropping the inertial terms on the left-hand side, results in the stress balance:

$$
\frac{\bar{F}_{z}}{\pi h^{2}}=\frac{1}{h}+\frac{\dot{h}^{2}}{2}-6 O h_{s} \frac{\dot{h}}{h}+\Delta \bar{\sigma}_{p} .
$$

In addition to the polymeric stress contribution $\Delta \bar{\sigma}_{p}$ [22], the expression contains also an inertial contribution $\dot{h}^{2} / 2$ that arises from the moving boundary of the free surface $[18,45]$. This can be understood by recognizing that in the actual axial force of Eq. (11)

$$
\frac{\bar{F}_{z}}{\pi h^{2}}=-\bar{P}+2 O h_{s} \frac{\partial \bar{v}}{\partial \bar{z}}+\bar{\sigma}_{p, z z}+\frac{2}{h},
$$

the nondimensional isotropic pressure $\bar{P}=P R_{0} / \gamma$ can be replaced by the radial components of the stress boundary condition at the free surface

$$
-\bar{P}+2 O h_{s} \frac{\partial \bar{v}}{\partial \bar{r}}+\bar{\sigma}_{p, r r}=-\frac{1}{h}+\frac{\dot{h}^{2}}{2} .
$$

The total axial force in Eq. (14) is thus composed of the bulk contributions of isotropic pressure and axial viscous and polymeric contributions, as well as a line tension term $2 / h$. It is the radial boundary condition that contains, in addition to the surface pressure $1 / h$, the inertial term $\dot{h}^{2} / 2$ arising from the movement of the boundary, as introduced in a similar manner by Tirtaatmadja et al. [18]. With the general relation of the axial and radial velocities to the extensional deformation rate for an incompressible cylindrical fluid filament of $\frac{\partial \bar{v}}{\partial \bar{z}}=-2 \frac{\partial \bar{v}}{\partial \bar{r}}=-2 \dot{h} / h$, inserting Eq. (16) into Eq. (15) directly results in Eq. (14) with the stress difference $\Delta \bar{\sigma}_{p}=\bar{\sigma}_{p, z z}-\bar{\sigma}_{p, r r}$, the characteristic Trouton ratio $\operatorname{Tr}=3$ that appears in the front factor 6 of the viscous term, and the radial thinning rates $\dot{\varepsilon} \tau=-2 \dot{h} / h$. The combination of the line tension $2 / h$ of Eq. (15) and the surface pressure $-1 / h$ of Eq. (16) results in the sign of the single term $1 / h$ in Eq. (14) as explained in [22].

In order to describe the evolution of the elastic stress term $\bar{\sigma}_{p, z z}$ in Eq. (14), Entov and Hinch [21] employed an elastic dumbbell constitutive model (FENE-P) to express the elastic 
nature of the dilute polymer solution [58]. The FENE model assumes that the polymer solution consists of a Newtonian solvent containing a dilute suspension of polymer chains that are modeled as nonlinear elastic springs with a maximum extensibility $L^{2}$. Entov and Hinch [21] have used this model to demonstrate that only the slowest relaxation mode of the entire molecule and the associated timescale is relevant in capillary breakup. We will, therefore, consider only a single relaxation mode in the following. The elastic deformation of the polymer coils is described with the conformation tensor $\mathbf{A}$, and the polymeric stress that is generated by deforming these dumbbells is expressed in tensorial form as

$$
\boldsymbol{\sigma}_{\mathbf{p}}=G(Z \mathbf{A}-\mathbf{I}) \text {. }
$$

The parameter $Z$ is the correction term accounting for the nonlinearity and the finite extensibility $L$ of the dumbbell as

$$
Z=\frac{L^{2}}{L^{2}-\operatorname{tr}(\mathbf{A})}
$$

with $\operatorname{tr}(\mathbf{A})$ being the trace of the conformation tensor.

The elastic deformation of the polymer coils under flow is correlated to the creation of polymeric stress by the microstructural evolution equation:

$$
\frac{D \mathbf{A}}{D t}-\mathbf{A} \nabla \mathbf{v}-\nabla \mathbf{v}^{T} \mathbf{A}=-\frac{1}{\lambda}(Z \mathbf{A}-\mathbf{I})
$$

that utilizes the upper-convected derivative to describe the confirmation changes in the dumbbell. Due to the radial symmetry and the uniaxial extensional flow field, the tensorial evolution equation [Eq. (19)] reduces to a set of two differential equations, one for the axial $\left(A_{z z}\right)$ and one for the radial $\left(A_{r r}\right)$ components:

$$
\begin{gathered}
\dot{A}_{z z}=-4 \frac{\dot{h}}{h} A_{z z}-\frac{1}{D e}\left(Z A_{z z}-1\right), \\
\dot{A}_{r r}=2 \frac{\dot{h}}{h} A_{r r}-\frac{1}{D e}\left(Z A_{r r}-1\right) .
\end{gathered}
$$

In these equations, the dotted symbols $\dot{A}_{z z}$ and $\dot{A}_{r r}$ represent the dimensionless time derivative of the respective polymer stretch, and the relaxation time is captured by the dimensionless Deborah number of Eq. (2).

Inserting the expression of Eq. (17) for the polymeric normal stress difference $\Delta \sigma_{p}=\sigma_{p, z z}-\sigma_{p, r r}$ in its dimensionless form into Eq. (14) results in the following expression for the stress balance:

$$
\frac{\bar{F}_{z}}{\pi h^{2}}=\frac{1}{h}+\frac{\dot{h}^{2}}{2}-6 O h_{s} \frac{\dot{h}}{h}+\operatorname{Ec} Z\left(A_{z z}-A_{r r}\right),
$$

where the scaling of the elastic modulus of the dilute solution with the initial capillary pressure returns again the dimensionless (global) elastocapillary number,

$$
E c=G R_{0} / \gamma .
$$

The dimensionless numbers for the 0.1 wt. \% PEO model solution are listed in Table $\mathrm{V}$ for both the CaBER and jetting experiments, where the latter values are calculated with
TABLE V. Dimensionless numbers of the CaBER and jetting experiments for the 0.1 wt. \% PEO solution.

\begin{tabular}{lcccc}
\hline \hline & $R_{0}(\mu \mathrm{m})$ & $O h_{s}$ & $D e$ & $E c$ \\
\hline CaBER & 1000 & 0.0040 & 0.445 & 0.0029 \\
Jetting & 85 & 0.0137 & 18.0 & 0.0346 \\
\hline \hline
\end{tabular}

$R=85 \mu \mathrm{m}$. The microstructural evolution equations (20) and (21) coupled with the stress balance (22) form a set of ODEs that can be solved to determine the evolution of the filament diameter in the CaBER experiment.

An elegant analytical solution for the evolution equation itself has been presented by Entov and Hinch [21], assuming that the axial stretch of the polymer chain is large $\left(A_{z z} \gg 1\right.$ $\left.\& A_{z z} \gg A_{r r}\right)$. This assumption implies that only Eq. (20) needs to be solved of the two in order to describe the increase of elastic stresses. Furthermore, they assumed that during the EC balance the deformation is still small compared to the maximal extension $\left(A_{z z} \ll L^{2}\right)$, so that the finite extensibility does not play a role and $Z \approx 1$. Both these assumptions refer, in principle, to the phenomenological description of the polymer deformation state in the third thinning regime in the discussion of Fig. 1(c). Under the assumption that the initial value of the axial stretch equals one $\left(A_{z z}^{0}=1\right)$, a solution for the polymeric stretch was derived as

$$
A_{z z}=\frac{1}{h^{4}} \exp \left(-\frac{\tau}{D e}\right) .
$$

Inserting this into Eq. (22) (with the required assumptions that $A_{z z} \gg A_{r r}$ and $Z \approx 1$ ) gives then a simplified stress balance for the third thinning regime of Fig. 1(c)

$$
\frac{\bar{F}_{z}}{\pi h^{2}}=\frac{1}{h}+\frac{\dot{h}^{2}}{2}-6 O h_{s} \frac{\dot{h}}{h}+\frac{E c}{h^{4}} \exp \left(-\frac{\tau}{D e}\right)
$$

that will be used in the following to obtain analytical solutions for the thinning dynamics of the CaBER or ROJER experiments in this regime.

\section{B. Tensile force of a viscoelastic filament in CaBER vs ROJER type experiments}

In order to determine the evolution of stresses and thinning dynamics of the filament from the stress balance of Eq. (14) [or Eqs. (22) or (25)], the (unknown) axial tension $F_{z}$ and its evolution needs to be determined. In the case of a uniform cylindrical viscous filament [so dropping inertial terms and polymeric contributions when integrating Eq. (11)], assuming that the bulk stresses in Eq. (14) are balanced by only the surface pressure, this axial tension $\bar{F}_{z}$ is equal to the line tension contribution $2 \pi h$ or dimensional $F_{z}=2 \pi \gamma R$ and thus linearly dependent on the filament radius $R$.

For a viscous filament generated by a CaBER experiment, the filament is connected to two fluid drops on the stationary end plates. These drops act as quasi-static reservoirs that soak up the fluid drained into them from the thinning filament. They also diminish the no-slip boundary condition at the end plates, which would otherwise induce a radial shear flow near the ends of the radially contracting fluid thread [59]. 
The curvature at the transition to the droplets disturbs uniformity and adds an extra contribution to the axial tension (in addition to the line tension), which can only be compensated in Eq. (14) by a change of the extension rate in the viscous term. McKinley et al. [45] showed that the observed thinning dynamics of a viscous filament do indicate a linear relation of the axial tension to the minimal radius, however, with a different front factor such that $F_{z}=2 X \pi \gamma R_{m}$. Furthermore, they showed that their observed factor was close to the one theoretically predicted by Papageorgiou [60] from a similarity analysis of the evolution of a viscous filament connected to spherical end drops, which gave $X=0.7127$.

For a viscoelastic liquid (that incorporates the polymeric stress), Entov and Hinch [21] assumed again a cylindrical filament but neglected the line tension term $h / 2$ in Eq. (15) and consequently set the total axial tension in the jet to be equal to the bulk contribution, which they assumed to vanish. While this gave the correct scaling of the filament thinning dynamics, Clasen et al. [22] showed later that for a quantitative description of the filament evolution the correct $F_{z}$ needs to be evaluated. They solved the stress balance with an asymptotical analysis in the center of the thread and on the end drops, and a complete expression for the tensile force is obtained with a similarity analysis in the corner region where the filament is attached to the end drops. This analysis showed that the tensile force in the filament for a CaBER experiment evolves as $\bar{F}_{z}=3 \pi h$ or

$$
F_{z}=3 \pi \gamma R_{m}
$$

so that axial tension is also linearly related to the filament diameter in the viscoelastic CaBER case, albeit again with a different front factor.

Both the analysis of Entov and Hinch [21] as well as the one from Clasen et al. [22] (and follow-up simulations by Bhat et al. [61] or Ardekani et al. [37]) that conclude in the axial force of Eq. (26) do not distinguish between a single liquid bridge and a continuous jet. We have, however, shown different exponential thinning dynamics between these two experimental setups in Sec. III, and will demonstrate in the following that this can be explained by a difference in the axial force in the jetting case compared to the single liquid bridge.

A different approach for acquiring an expression for the tensile force in a jet was already suggested in the research on the gobbling phenomenon [39]. Following this, we cannot evaluate the tensile force solely based on a single Lagrangian element, but have to consider the entire jet.

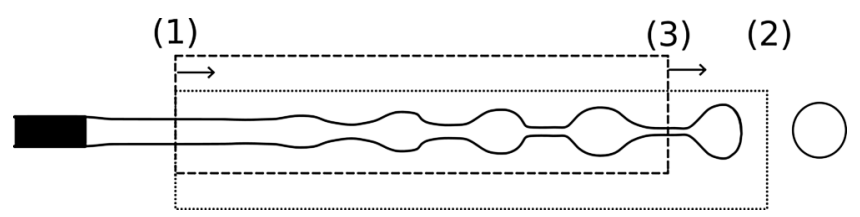

FIG. 10. Control volumes of the viscoelastic jet. Dotted line: control volume with stationary boundaries in the uniform part of the jet (1) and after the last connected drop (2). Dashed line: control volume with boundaries moving with the velocity of the jet in the uniform part (1) and the midfilament point between two asperities forming on the jet (3).
The axial force in a jet can be estimated by integrating the one-dimensional momentum equation [Eq. (11)] over a control volume that is shown in Fig. 10. One boundary of the control volume is located at a stationary location right below the point where the jet is attached to the nozzle, so in the stable section where the jet is still uniform [indicated as (1)]. The other boundary is positioned right after the final droplet forming on the jet [indicated as (2)]. The integrated equation is given by

$$
\begin{aligned}
& \frac{\partial}{\partial \tau}\left(\int_{\bar{z}_{1}}^{\bar{z}_{2}}\left(h^{2} \bar{v}\right) d \bar{z}\right)+\left.h^{2} \bar{v}^{2}\right|_{\bar{z}_{1}} ^{\bar{z}_{2}} \\
& \quad=\left.h^{2}\left(K+3 O h_{s} \frac{\partial \bar{v}}{\partial \bar{z}}+\Delta \bar{\sigma}_{p}+\frac{\dot{h}^{2}}{2}\right)\right|_{\bar{z}_{1}} ^{\bar{z}_{2}}=\left.\frac{\bar{F}_{z}}{\pi}\right|_{\bar{z}_{1}} ^{\bar{z}_{2}} .
\end{aligned}
$$

By averaging this equation over a time interval that is substantially longer than the time between two beads passing across any given cross section of the jet, the first term on the lefthand side can be dropped. Moreover, following the approach of Clanet and Lasheras [62] for describing the momentum loss in a jet, the time averaging implies that the convective term can be rewritten in function of the flow rate $\bar{Q}=\bar{v} h^{2}$ through the jet into the control volume at (1), and the detaching droplets leaving at (2)

$$
h^{2} \bar{v}_{\bar{z}_{1}}^{\bar{z}_{2}}=\frac{\bar{Q} \bar{v}}{\pi}-\frac{\bar{Q} \bar{v}_{d r o p}}{\pi} .
$$

This change in momentum of the detaching droplets is balanced by the net force on the control volume. As the connectivity between droplets at (2) is lost, the tension across the boundary at (2) is zero, so that the force on the right-hand side of Eq. (27) is just

$$
\left.\frac{\bar{F}_{z}}{\pi}\right|_{\bar{z}_{1}} ^{\bar{z}_{2}}=h^{2}\left(K+3 O h_{s} \frac{\partial \bar{v}}{\partial \bar{z}}+\Delta \bar{\sigma}_{p}+\frac{\dot{h}^{2}}{2}\right)-0 .
$$

Since in this uniform section of the jet at (1) the inertial, viscous, and elastic contribution on the right-hand side of Eq. (30) can be neglected, and since the straight jet shape implies $h^{2} K=1$, it follows that the net force on the control volume and thus the tension in the jet at (1) is

$$
\frac{\bar{F}_{z}}{\pi}=1
$$

or in dimensional form

$$
F_{z}=\pi R_{0} \gamma .
$$

The velocity of the jet within the control volume remains constant up to the last connected drop. This can be experimentally observed from the constant jet diameter in the initial, uniform part of the jet, as well as from the constant velocity of the Lagrangian asperities that develop eventually into the droplets, and the minimum filament radius in-between. Figure 11 shows exemplarily such a constant velocity of the asperities along the jet. It is only the last connected drop that experiences a deceleration to $\bar{v}_{\text {drop }}$ from the constant tension in the remaining filament, up to the point where the connecting filament breaks. A constant 


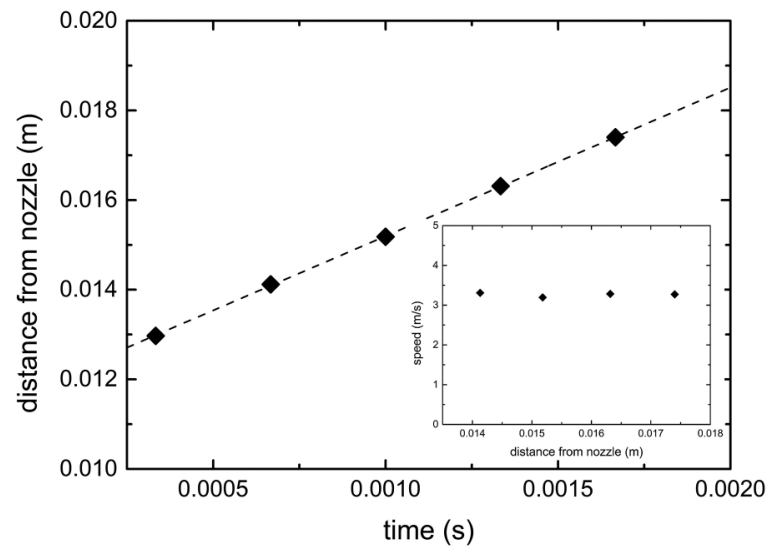

FIG. 11. Position of asperities or forming droplets as a function of time for a jetting experiment of a PEO solution of $1000 \mathrm{~kg} / \mathrm{mol}, \mathrm{c}=0.001 \mathrm{wt} . \%$, in water-glycerol $60-40$. The insert gives the velocity of the droplet at each position along the jet.

velocity of asperities/droplets along the jet, as well as a deceleration of only the final droplet has also been observed by Clasen et al. [39] for lower Weber numbers and jet velocities close to the jetting/dripping transition. In their case, the low velocities caused the final drop not only to be decelerated by the constant tension, but also to be reaccelerated towards the nozzle, while still maintaining a constant velocity along the jet.

This constant velocity of the jet and asperities allows to draw a second control volume (dashed line in Fig. 10) where the first boundary position (1) is the same as for the previous case, but where the other boundary (indicated as (3) in Fig. 10) is located at a minimum between two droplets, and where both boundaries move now with the same constant velocity of the Lagrangian fluid elements at (1) and (3). In this case since there is no momentum in- or out-flux, the net force from Eq. (27) on this control volume is zero. Since this implies that the tensile forces at the boundaries (1) and (3) are balanced, and since Eq. (30) showed that the tensile force in the uniform part of the jet at (1) is constant, the tensile force at the minimum (3) will be constant at any position of the minimum along the jet, and equal to $\pi R_{0} \gamma$.

\section{Analytical solutions for the thinning dynamics in a CaBER type experiment}

Inserting now for the CaBER case the force of Eq. (26) in its dimensionless form $\bar{F}_{z}=3 \pi h$ into the stress balance of Eq. (25) gives

$$
\frac{3}{h}=\frac{1}{h}+\frac{\dot{h}^{2}}{2}-6 O h_{s} \frac{\dot{h}}{h}+\frac{E c}{h^{4}} \exp \left(-\frac{\tau}{D e}\right) .
$$

With the assumption that, once elastic contributions grow sufficiently large, the inertial and viscous term can be neglected, the stress balance reduces to the EC balance:

$$
\frac{2}{h}=\frac{E c}{h^{4}} \exp \left(-\frac{\tau}{D e}\right) \text {. }
$$

Solving this for the radius finally yields:

$$
h=\left(\frac{E c}{2}\right)^{1 / 3} \exp \left(-\frac{\tau}{3 D e}\right) .
$$

This is the result that Clasen et al. [22] obtained, which is, apart from the prefactor of $2^{-1 / 3}$ [that arises when using the correct force of Eq. (26)], equivalent to the original solution of Entov and Hinch [21]. Furthermore, this is also equivalent to Eq. (5) that was used to process the CaBER experiments. According to this expression, the ligament radius will only reach zero at an infinite time, since the finite extensibility of the polymer molecules is not taken into account. Entering Eq. (34) into Eq. (24), the elastic stretch as well as the polymeric stress appear to be growing unrestrictedly. However, when the axial stretch $A_{z z}$ approaches the squared finite extensibility parameter $L^{2}$, the nonlinear correction term $Z$ will lead to an upper bound of the polymeric stress and the finite extension will determine the final breakup of the filament.

\section{Elastic balance in the ROJER}

To assess the thinning rate of the jet in the elasticity dominated regime, the expression for the constant force of Eq. (31) in its dimensionless form $\bar{F}_{z}=\pi$ inserted into the stress balance of Eq. (25) generates the stress balance for the viscoelastic jet:

$$
\frac{1}{h^{2}}=\frac{1}{h}+\frac{\dot{h}^{2}}{2}-6 O h_{s} \frac{\dot{h}}{h}+\frac{E c}{h^{4}} \exp \left(-\frac{\tau}{D e}\right) .
$$

This stress balance is different from the balance for the gobbling phenomenon [39], since the inertial term is taken into account instead of only the viscous dissipation. Different from the stress balance for the capillary breakup of a single bridge of Eq. (32), an additional term $1 / h^{2}$ appears in the stress balance originating from the constant force along the jet. This constant force will grow increasingly important close to breakup as the area of the liquid column rapidly reduces.

Similar to the analysis for the CaBER experiment, we can first simplify the stress balance in this regime by neglecting the inertial and viscous term to

$$
\frac{1}{h^{2}}=\frac{1}{h}+\frac{E c}{h^{4}} \exp \left(-\frac{\tau}{D e}\right) .
$$

Furthermore, approaching small filament dimensions also the capillary pressure term $1 / h$ in Eq. (35) becomes subdominant in comparison to the now constant force term $1 / h^{2}$, so that the dominant balance is established between the axial stress and the elastic stress (called "AE balance" in the following):

$$
\frac{1}{h^{2}}=\frac{E c}{h^{4}} \exp \left(-\frac{\tau}{D e}\right)
$$

While it is initially unintuitive not to directly have a Laplace pressure term $1 / h$ in the balance that drives the capillary thinning, it should be noted that is still the line tension of the jet that sets the constant tension that is eventually balancing polymer elasticity. Solving now for the radius evolution of 

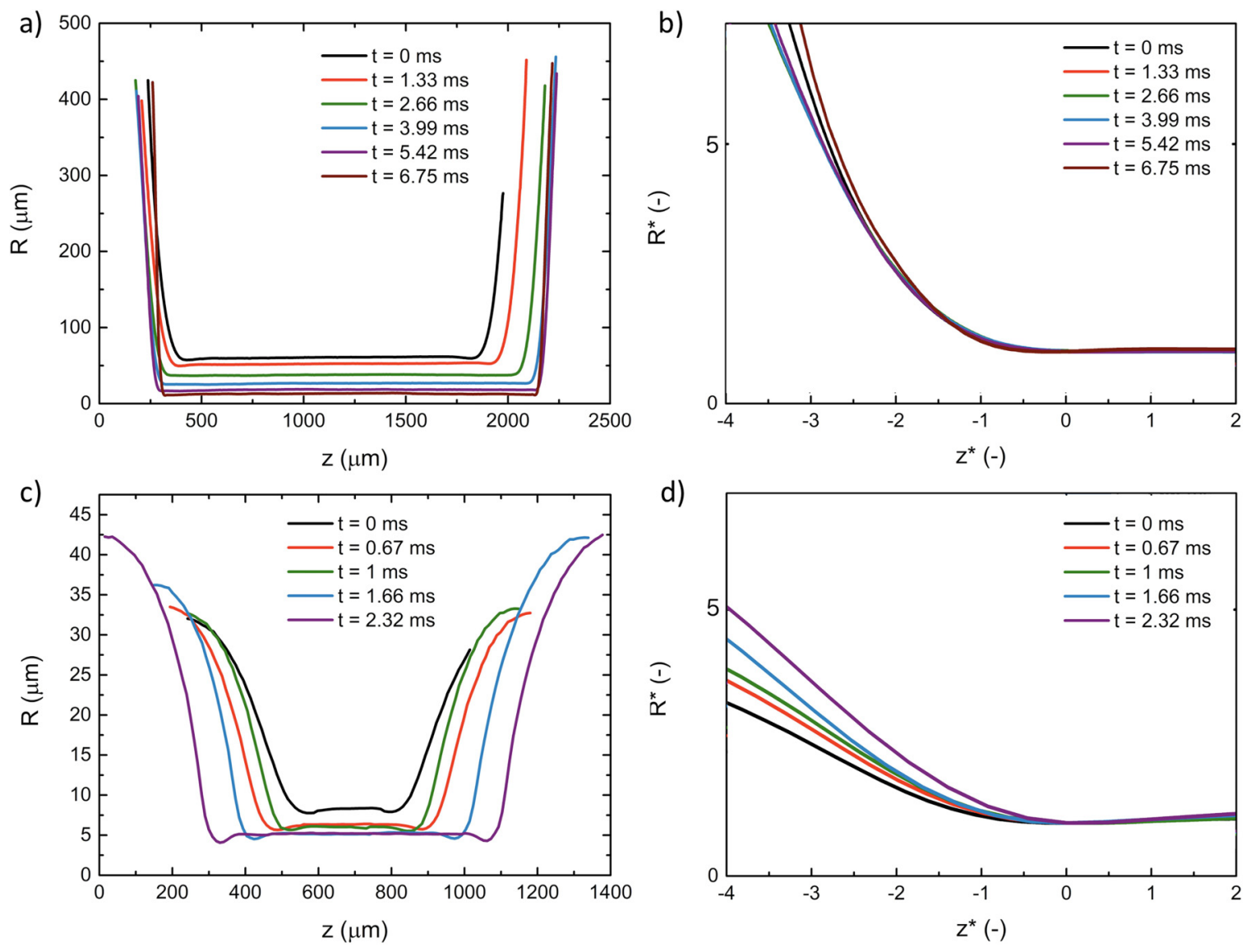

FIG. 12. Experimental filament profiles of the $0.005 \mathrm{wt} \%$ PEO solution of $M_{w}=1000 \mathrm{~kg} / \mathrm{mol}$ in water/glycerol $60 / 40$ during the different free-surface experiments, with the times for each profile indicated in the legend. The profiles $R$ are shown as a function of the axial coordinate $z$ for the (a) CaBER and (c) excited jetting experiments, respectively. The rescaled edge profiles of the corner region exhibit a similar shape for (b) the CaBER experiments, whereas (d) the rescaled jetting profiles of a ROJER type experiment does not show the same self-similarity.

the jet during the $\mathrm{AE}$ balance regime one obtains

$$
h=\sqrt{E c} \exp \left(-\frac{\tau}{2 D e}\right) .
$$

This is the same expression that was found for the gobbling phenomenon, where the viscous term was neglected in the stress balance instead of the inertial term [39]. Like in the previous analysis for the CaBER, the radius decreases exponentially in the elasticity dominated regime, however, the time scale in the $\mathrm{AE}$ balance regime is different from the EC balance. The creation of new surface near the nozzle generates a constant tensile force in the jet, which changes the thinning dynamics. The introduction of this constant force into the stress balance changes the dimensionless time scale from $3 D e$ to $2 D e$, which is the value that was observed throughout all jetting experiments.

Verifying a different tensile force during jetting is challenging (compared to during a CaBER experiment, which has been done by Willenbacher and co-workers [63]). However, we can analyze the shape of the corner region, where a filament is attached to the neighboring drops. When the tensile force decreases exponentially in time, as it does for a single liquid bridge since $F_{z}(t) \sim R_{m}(t)$, simulations and experiments have shown that this corner should reveal a self-similar structure $[22,61]$. To examine the existence of this similarity region, all lengths are scaled with a characteristic length scale, the minimal radius $R_{m}$, giving

$$
\begin{gathered}
R^{*}=\frac{R}{R_{m}}, \\
z^{*}=\frac{z-z_{0}}{R_{m}} .
\end{gathered}
$$

The axial origin $z_{0}$ is asymptotically located in the corner region. These rescaled free-surface profiles are shown next to the original profiles in Figs. 12(a) and 12(b) for CaBER capillary breakup experiments of the $0.1 \mathrm{wt}$ \% PEO solutions. The experimental profiles converge in both cases onto a master curve with increasing thinning time, demonstrating the self-similar structure of the corner. This suggests that the tensile force is exponentially decreasing as expected for the CaBER experiment.

Also the profiles of an excited jet experiment on the same solution are rescaled, using the same scales. To obtain sufficient resolution in the corner region, the thinning jet was visualized with the same microscopic objective that was used for the CaBER experiments. Due to the limited field of view of this setup, a low Weber number of $W e=2.9$ was chosen to be able follow a sufficient number of instabilities within the observation window. Figure 12(c) shows a sequence of free- 
a)
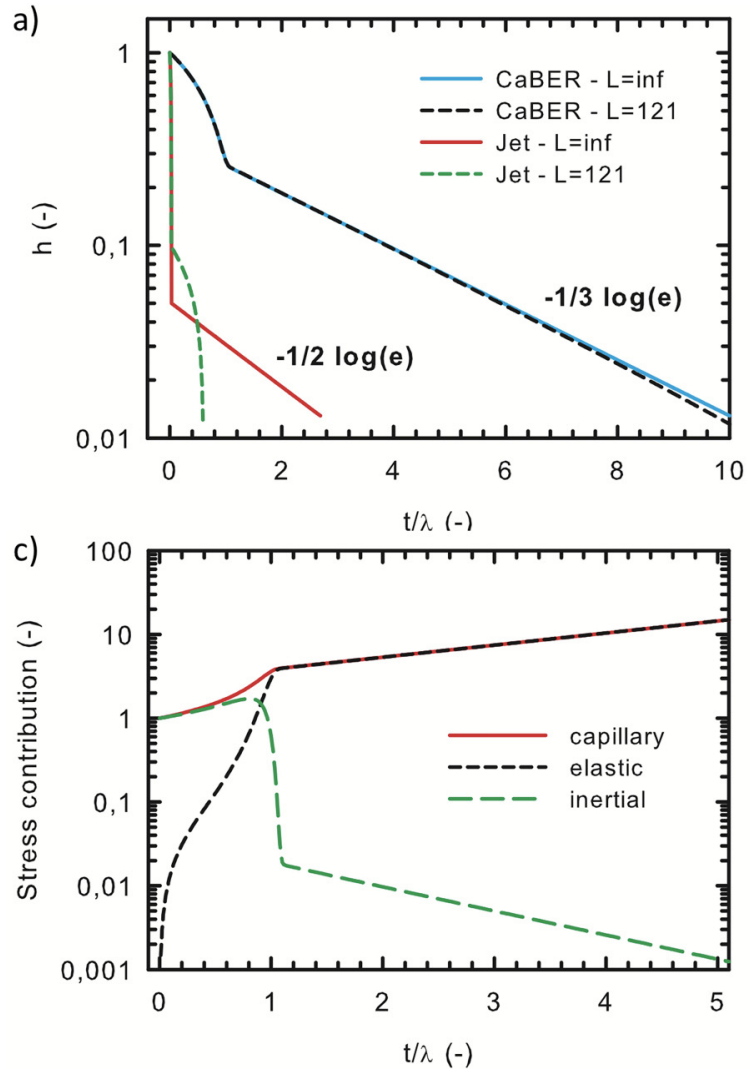

b)

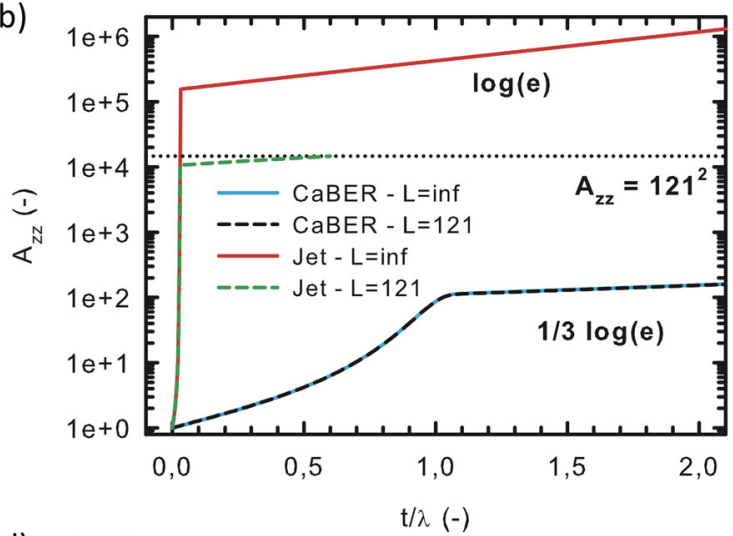

d)

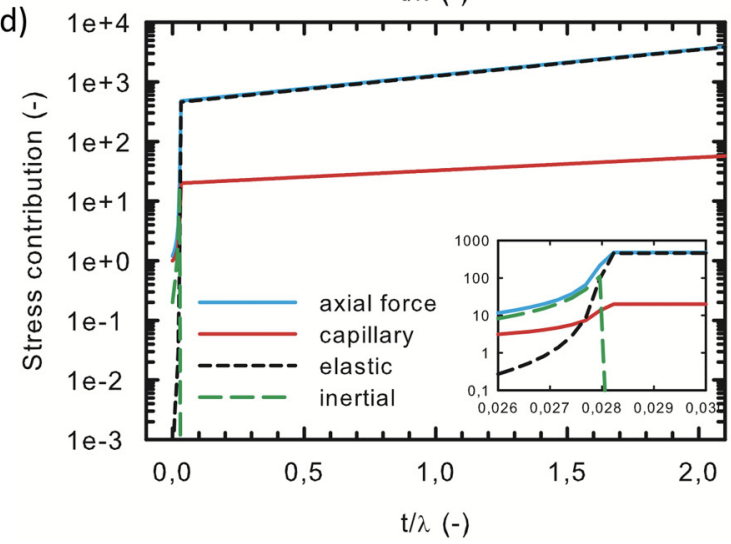

FIG. 13. Numerical simulation of capillary breakup in the jetting and CaBER setup with the dimensionless parameters listed in Table V. (a) The evolution of the filament radius, (b) the axial stretch of the polymer chains and the evolution of the stresses for $L=\infty$ in (c) the CaBER and (d) the jetting setup, in which the insert focusses on the transition from the IC to the elastic balance regime.

surface profiles of the jet. The consecutive corner regions are rescaled in Fig. 12(d) and do not form a master curve. The absence of a similarity region for both jetting experiments suggests a different tensile force in the thread that does not decrease exponentially in time as in the CaBER experiments, and leads to different thinning rates in both experiments.

\section{E. Numerical simulations}

The combination of the full stress balance of Eq. (22) with the evolution equation [Eqs. (20)-(21)] (and the respective axial force for either the CaBER or the ROJER case) makes an initial value problem that can be used to describe the complete thinning of the inertia-elastic filament. This simple model is used to explore the transitions between different thinning regimes in both CaBER and jetting flows. By solving the microstructural evolution equation, the growth of the axial stretch $A_{z z}$ provides insight into the polymer deformation in the uniaxial extensional flow field. The equations are solved in MATLAB with an implicit BDF-solver (routine ode15s) to cope with the stiffness of the system. The transition from the inertia to the elasticity dominated regime causes an abrupt change in the polymeric deformation, so the absolute and relative tolerances are chosen at a low value of $10^{-10}$. It should be noted that the approach for these simulations is different from earlier works [19], as it explicitly includes the inertial term $\dot{h}^{2} / 2$ in Eq. (22), which is necessary in order to capture the initial thinning regime before the onset of the exponential thinning regime, which is dominated by inertia (as indicated by the low global Ohnesorge numbers in Tables I and V).

A first set of results of the numerical simulation for both setups are presented in Fig. 13, using an ideal state of undeformed polymer coils as the initial conditions at the start of the experiments, so that the initial value of the three dimensionless variables are all equal to one: $h^{0}=1, A_{z z}^{0}=1$, and $A_{r r}^{0}=1$. A reference radius $R_{0}$ is chosen to resemble the experimental observation and, therefore, the dimensionless groups in Table V are used for the calculations. Fig. 13(a) compares the evolution the dimensionless filament radius $h$ as a function of time, which has been scaled with the relaxation time to emphasize the different time scales in the exponential thinning regime. When finite extensibility effects are neglected $(L=\infty)$, the time scales of $3 \lambda$ and $2 \lambda$ are recovered over a long period for the CaBER and ROJER type experiment, respectively, in agreement with the experimental observations and the analytical derivation in Secs. IV C and IV D.

For the CaBER simulations, the transition for the IC to an EC regime can be explained by the temporal evolution of the stress contributions in Eq. (33) that is shown in Fig. 13(c). Initially, the polymer chains are close to the equilibrium conformation and the inertial acceleration balances the capillary pressure in the fluid column. This balance results in a fast decay of the radius, inducing strong stretching of the polymer as shown in Fig. 13(b). The elastic stress in the column rises quickly to balance the capillary pressure and the inertial 

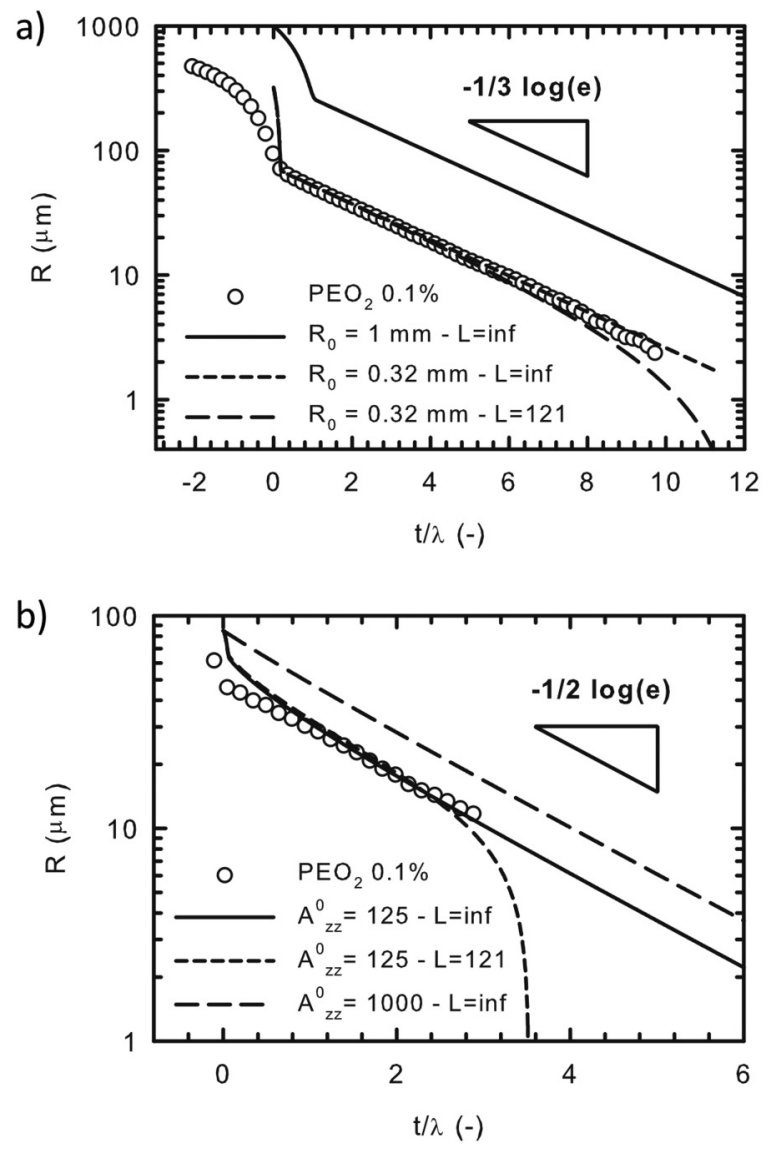

FIG. 14. Comparison of the experimental evolution of the minimal radii with numerical simulations. (a) For the CaBER setup, an agreement between experiment and model is found by lowering the initial radius $R_{0}$. (b) The jetting simulations are matched to the experimental observations by changing the initial axial stretch $A_{z z}^{0}$ of the polymer molecules.

contribution drops to a negligible value. As a result of this EC balance, the growth of the axial deformation of the chains suddenly slows down to an exponential increase with a time constant corresponding to three times the relaxation time of the fluid: $A_{z z} \sim \exp (t / 3 \lambda)$.

The respective elastic and capillary stresses in Fig. 13(c) match this exponential increase. If the finite extensibility effects are included in the analysis, at later times a deviation from the exponential decrease of the filament radius is observed in Fig. 13(a) at $h \approx 0.02$, which corresponds to an axial stretch $A_{z z} \approx 0.1 L^{2}$. As the axial deformation approaches the finite extensibility parameter, the nonlinear correction term $Z$ increases considerably, resulting in a sudden breakup.

The numerical simulations for the CaBER are also compared with experimentally obtained filament radii in Fig. 14. The model solution for the CaBER case using $R_{0}=1 \mathrm{~mm}$ displays a very similar shape as the experimental curve, however, the onset of the EC regime occurs at a higher radius. This inconsistency in the transition radius was also encountered in previous studies $[18,64]$ : the IC regime typically demonstrates necking near the end droplets and cannot be described with a zero-dimensional model that furthermore neglects the gravitational component in Eq. (11) in the initial regime where in the CaBER case the Bond number is still close to unity. Furthermore, recent studies by Prabhakar and co-workers $[65,66]$ showed that stretch-induced changes in hydrodynamic screening will affect the thinning dynamics even before the onset of the coil-stretch transition as described by our simplified model. A better agreement of 0-D models to the experimental EC regime is usually found by choosing a smaller value for the reference radius $R_{0}$ as done in Fig. 14(a). While decreasing the reference radius results in a poor description of the inertia-capillary regime, it captures the thinning dynamics in the EC regime well.

In the jetting simulations of a ROJER type experiment in Fig. 13(a), again using undeformed polymer coils as an initial state, the filament radius decays initially much faster in comparison to the CaBER case before reaching an exponential thinning regime, and also initially much faster than what is experimentally observed for the ROJER experiments, for example, in Fig. 5. This fast decay originates from the different stress balance of which the evolution of the different relevant terms is shown in Fig. 13(d). The term driving the filament breakup is the stress from the constant axial force as indicated in Eq. (36). This term scales with the inverse squared radius and, therefore, increases more quickly than the capillary stress, and it is thus this contribution that balances the inertial stresses as can be seen in the inset of Fig 5 (d). Moreover, this larger axial driving stress requires the polymer chains to be stretched much further from their initial undeformed state to produce sufficient elastic stress to eventually balance this axial stress. When this happens, Fig. 13(b) shows that at this point the axial deformation of the initially undeformed chains is 3 orders of magnitude larger than at the onset of the EC balance in the CaBER case. Still, once this balance is reached, the axial stretch and the elastic stress increase exponentially when $L=\infty$, and with the experimentally observed smaller time constant: $A_{z z} \sim \exp (t / \lambda)$. However, including finite extensibility effects in the jetting experiment, an exponential decrease of the filament radius is not observed. As can be seen in Fig. 13(b) when setting $L=121$ for the jetting case, the initial polymer deformation $A_{z z}$ in the inertial regime is already close to the maximum stretch $L^{2}$, so that it is directly followed by a nonlinear elastic stress buildup, leading to a very fast breakup of the filament.

These two discrepancies of the ROJER simulations in comparison to experiments, the too late onset of the elastic balance regime and the too strong deformation of the polymer at this onset to observe an exponential thinning, indicate that the initial assumption of undeformed polymer coils is not correct. Indeed, a deformation of the polymer coils when passing through the contraction flow at the nozzle entrance, as well as in the relatively high shear rates experienced inside the nozzle, that persists in the jet when exiting the nozzle, has been previously discussed for jet thinning dynamics of polymer solutions [39,67]. Furthermore, it is probable that the polymer chains are partially oriented and elongated after exiting the nozzle. At this point, the velocity field in the jet undergoes an abrupt change from a Poiseuille flow inside the tube to a plug flow in the initial straight section of the jet. This stick-slip singularity at the nozzle exit results in potentially large initial elastic stresses in the jet 
[68]. Since the Deborah number in Table V is sufficiently large $(D e \gg 1)$, effects of this initial configuration can propagate along the jet while the fluid column begins to exhibit the linear instabilities [39].

Such a pre-stretch at the nozzle exit can be included in the jetting model by changing the initial chain confirmation $\left(A_{z z}^{0}>1, A_{r r}^{0}=A_{z z}^{0-1 / 2}\right)$. By incorporating this additional elastic contribution, the initial axial stress in the jet is

$$
\frac{F_{z}}{\pi R_{0}^{2}}=\frac{\gamma}{R_{0}}+E c Z\left(A_{z z}^{0}-A_{z z}^{0^{-1 / 2}}\right) .
$$

The initial conormation $A_{z z}^{0}$ and the associated initial stress are new initial values for the numerical simulations of the coupled differential equations for the jet [Eqs. (20), (21), (22), and the constant axial force $\left.\bar{F}_{z}=\pi\right]$. $A_{z z}^{0}$ becomes thus an extra parameter to fit the experimental observations. The results for ROJER simulations that include a predeformation of the polymers at the nozzle are compared to experimental thinning data in Fig. 14(b). By employing a larger pre-stretch $A_{z z}^{0}=1000$, an axial-elastic balance is immediately established at the initial radius and the appearance of an initial inertia-capillary thinning and fast initial decay of the filament radius is suppressed. A more consistent description is acquired by reducing the initial axial stretch to $A_{z z}^{0}=125$. The modeled radius shows a more gradual drop from the initial value and subsequently approaches the experimental data points. The driving axial stress is not solely balanced by the elastic stress for times $t<2 \lambda$, leading to a fairly long transition zone where the capillary term still affects the thinning. The elastic stress only matches the driving stress after this period and then an exponential decrease of the filament radius is retrieved. It should be noted that time scale observed in the exponential $\mathrm{AE}$ regime is in all cases the new time scale of two times the relaxation time, independent of the predeformation value for $A_{z z}^{0}$, and matching the experimental radius decay.

During the $\mathrm{AE}$ balance, the polymer chains are still stretched at a faster rate than in the CaBER case [see Fig. 13(b)]. Hence breakup occurs slightly sooner than in the experiments when finite extensibility effects are included in the model. However, in both the CaBER and the jetting case, the finite extensibility of the polymer chains affects the thinning dynamics sooner in the simulations than in the experiments. A comparable difference between the modeled and experimental finite extensibility effects is also encountered in other studies on capillary breakup of similar polymer solutions [69,70]. Two possible explanations have been proposed for the earlier onset of these finite extensibility effects. The former assumes a difference between the theoretical finite extensibility $L$ that was calculated based on molecular parameters [see Eq. (7)] and the experimentally observed $L$ [71]. The latter states that pre-averaging of the connector vector $\mathbf{Q}$ in the Peterlin approximation of the FENE model overpredicts the elastic stresses in a uniaxial extensional flow compared to the full FENE model [72].

\section{CONCLUSION}

In this paper, we have investigated the instability growth and the resulting breakup of weakly viscoelastic jets with low Ohnesorge numbers. Jetting experiments were executed for three model solutions over a range of Weber numbers from the dripping-jetting transition at $W e=O(1)$ to long stable jets at $W e=80$, which corresponds to the experimental limit of our setup. We have focused on this important elasticity controlled thinning regime where the minimal radius of the instability decreases exponentially in time. The thinning dynamics during this regime are compared with CaBER capillary breakup experiments of single liquid filaments, and a different time scale for the exponential decay is identified for both free-surface flows. Whereas the generally employed time scale $\theta=3 \lambda$ is observed for the CaBER experiments, the jetting experiments exhibit a time scale $\theta=2 \lambda$, which was previously only suggested for jets in the vicinity of the dripping to jetting transition. This new scaling remains valid for all examined Weber numbers $(W e>1)$.

Both time scales are explained with a stress balance over the viscoelastic filament. The different scaling in the jet is attributed to a constant axial force in the jet, which is caused by the creation of new surface under the nozzle. This results for the jet in a balance of the constant axial force and elasticity (AE balance) during the exponential thinning regime, in contrast to an EC balance in the CaBER experiment in this regime. Numerical simulations of the jet breakup with the FENE model are in agreement with the experimental observation by selecting a proper value for the initial deformation of the polymer molecules $A_{z z}^{0}$. Additionally, the experimental profiles of the corner region where the filament is connected to the droplet are rescaled according to the scales proposed by Clasen et al. [22]. The self-similar structure present in the CaBER experiments is not observed during the jetting experiments, indicating that the axial force is indeed not decaying with the filament radius. The existence of a different scaling in the exponential thinning regime in a continuous jet is not only of fundamental interest, but it also strongly affects the result interpretation of new jetting rheometers as the ROJER [36], resulting in a 50\% increase of the extracted relaxation time.

\section{ACKNOWLEDGMENTS}

W.M. would like to financial support from the ERC-2007-StG starting Grant No. 203043 NANOFIB. C.M. and O.G.H. would like to acknowledge support from the UK Engineering and Physical Sciences Research (EPSRC) under Grant No. EP/H018913/1, and C.C. would like to acknowledge support from the Research Foundation Flanders (FWO, project G077916N).

\section{References}

[1] Eggers, J., "Nonlinear dynamics and breakup of free-surface flows," Rev. Mod. Phys. 69, 865-929 (1997).

[2] Eggers, J., and E. Villermaux, "Physics of liquid jets," Rep. Prog. Phys. 71, 036601 (2008).

[3] Kroesser, F., and S. Middleman, "Viscoelastic jet instability," AICHE J. 15, 383-386 (1969). 
[4] Goldin, M., J. Yerushalmi, R. Pfeffer, and R. Shinnar, "Breakup of a laminar capillary jet of a viscoelastic fluid," J. Fluid Mech. 38, 689-711 (1969).

[5] Middleman, S., "Stability of a viscoelastic jet," Chem. Eng. Sci. 20, 1037-1040 (1965).

[6] Schummer, P., and K. H. Tebel, "A new elongational rheometer for polymer-solutions," J. Non-Newtonian Fluid Mech. 12, 331-347 (1983).

[7] Yarin, A., Free Liquid Jets and Films: Hydrodynamics and Rheology (Longman Scientific \& Technical, London, 1993).

[8] C. Clasen, P. M. Phillips, L. Palangetic, and J. Vermant, "Dispensing of rheologically complex fluids: The map of misery," AICHE J. 58, 3242-3255 (2012).

[9] Christanti, Y., and L. M. Walker, "Surface tension driven jet break up of strain-hardening polymer solutions," J. Non-Newtonian Fluid Mech. 100, 9-26 (2001).

[10] Morrison, N. F., and O. G. Harlen, "Viscoelasticity in inkjet printing," Rheol. Acta 49, 619-632 (2010).

[11] Mun, R. P., J. A. Byars, and D. V. Boger, "The effects of polymer concentration and molecular weight on the breakup of laminar capillary jets," J. Non-Newtonian Fluid Mech. 74, 285-297 (1998).

[12] de Gans, B. J., P. C. Duineveld, and U. S. Schubert, "Inkjet printing of polymers: State of the art and future developments," Adv. Mater. 16, 203-213 (2004).

[13] Derby, B., "Inkjet printing of functional and structural materials: Fluid property requirements, feature stability, and resolution," Annu. Rev. Mater. Res. 40, 395-414 (2010).

[14] Vadillo, D. C., T. R. Tuladhar, A. C. Mulji, S. Jung, S. D. Hoath, and M. R. Mackley, "Evaluation of the inkjet fluid's performance using the "cambridge trimaster" filament stretch and break-up device," J. Rheol. 54, 261-282 (2010).

[15] Bhat, P. P., S. Appathurai, M. T. Harris, M. Pasquali, G. H. McKinley, and O. A. Basaran, "Formation of beads-on-a-string structures during break-up of viscoelastic filaments," Nat. Phys. 6, 625-631 (2010).

[16] Chen, Y. J., and P. H. Steen, "Dynamics of inviscid capillary breakup: Collapse and pinchoff of a film bridge," J. Fluid Mech. 341, 245-267 (1997).

[17] Day, R. F., E. J. Hinch, and J. R. Lister, "Self-similar capillary pinchoff of an inviscid fluid," Phys. Rev. Lett. 80, 704-707 (1998).

[18] Tirtaatmadja, V., G. H. McKinley, and J. J. Cooper-White, "Drop formation and breakup of low viscosity elastic fluids: Effects of molecular weight and concentration," Phys. Fluids 18, 043101 (2006).

[19] Clasen, C., J. P. Plog, W. M. Kulicke, M. Owens, C. Macosko, L. E. Scriven, M. Verani, and G. H. McKinley, "How dilute are dilute solutions in extensional flows?," J. Rheol. 50, 849-881 (2006).

[20] Bousfield, D. W., R. Keunings, G. Marrucci, and M. M. Denn, "Nonlinear-analysis of the surface-tension driven breakup of viscoelastic filaments," J. Non-Newtonian Fluid Mech. 21, 79-97 (1986).

[21] Entov, V. M., and E. J. Hinch, "Effect of a spectrum of relaxation times on the capillary thinning of a filament of elastic liquid," J. NonNewtonian Fluid Mech. 72, 31-53 (1997).

[22] Clasen, C., J. Eggers, M. A. Fontelos, J. Li, and G. H. McKinley, "The beads-on-string structure of viscoelastic threads," J. Fluid Mech. 556, 283-308 (2006).

[23] Plog, J., W.-M. Kulicke, and C. Clasen, "Influence of the molar mass distribution on the elongational behaviour of polymer solutions in capillary break-up, case study: Elongational behaviour of blended cellulose ethers," Appl. Rheol. 15, 28-37 (2005).

[24] Yesilata, B., C. Clasen, and G. McKinley, "Nonlinear shear and extensional flow dynamics of wormlike surfactant solutions," J. NonNewtonian Fluid Mech. 133, 73-90 (2006).
[25] Miller, E., C. Clasen, and J. Rothstein, "The effect of step-stretch parameters on capillary breakup extensional rheology (caber) measurements," Rheol. Acta 48, 625-639 (2009).

[26] Regev, O., S. Vandebril, E. Zussman, and C. Clasen, "The role of interfacial viscoelasticity in the stabilization of an electrospun jet," Polymer 51, 2611-2620 (2010).

[27] Clasen, C., "Capillary breakup extensional rheometry of semi-dilute polymer solutions,” Korea-Aust. Rheol. J. 22, 331-338 (2010).

[28] Erni, P., M. Varagnat, C. Clasen, J. Crest, and G. McKinley, "Microrheometry of sub-nanoliter biopolymer samples: NonNewtonian flow phenomena of carnivorous plant mucilage," Soft Matter 7, 10889 (2011).

[29] Rodd, L. E., T. P. Scott, J. J. Cooper-White, and G. H. McKinley, "Capillary break-up rheometry of low-viscosity elastic fluids," Appl. Rheol. 15, 12-27 (2005).

[30] Campo-Deaño, L., and C. Clasen, "The slow retraction method (SRM) for the determination of ultra-short relaxation times in capillary breakup extensional rheometry experiments," J. Non-Newtonian Fluid Mech. 165, 1688-1699 (2010).

[31] Vadillo, D. C., W. Mathues, and C. Clasen, "Microsecond relaxation processes in shear and extensional flows of weakly elastic polymer solutions," Rheol. Acta 51, 755-769 (2012a).

[32] De Dier, R., W. Mathues, and C. Clasen, "Extensional flow and relaxation of semi-dilute solutions of schizophyllan," Macromol. Mater. Eng. 298, 944-953 (2013).

[33] Mathues, W., C. McIlroy, O. Harlen, and C. Clasen, "Capillary breakup of suspensions near pinch-off," Phys. Fluids 27, 093301 (2015).

[34] Christanti, Y., and L. M. Walker, "Effect of fluid relaxation time of dilute polymer solutions on jet breakup due to a forced disturbance," J. Rheol. 46, 733-748 (2002).

[35] Sharma, V., A. Ardekani, and G. H. McKinley, "Beads on a string' structures and extensional rheometry using jet break-up," 5th Pacific Rim Conference on Rheology, Sapporo, Japan, 2010.

[36] Keshavarz, B., V. Sharma, E. Houze, M. Koemer, J. Moore, P. Cotts, P. Threfrall-Holmes, and G. H. McKinley, "Studying the effects of elongational properties on atomization of weakly viscoelastic solutions using Rayleigh Ohnesorge jetting extensional rheometry (ROJER)," J. Non-Newtonian Fluid Mech. 222, 171-189 (2015).

[37] Ardekani, A. M., V. Sharma, and G. H. McKinley, "Dynamics of bead formation, filament thinning and breakup in weakly viscoelastic jets," J. Fluid Mech. 665, 46-56 (2010).

[38] Sharma, V., S. Haward, J. Serdy, B. Keshavarz, A. Soderlund, P. Threfrall-Holmes, and G. H. McKinley, "The rheology of aqueous solutions of ethyl hydroxy-ethyl cellulose (ehec) and its hydrophobically modified analogue (hmehec): extensional flow response in capillary break-up, jetting (ROJER) and in a cross-slot extensional rheometer," Soft Matter 11, 3251-3270 (2015).

[39] Clasen, C., J. Bico, V. M. Entov, and G. H. McKinley, "'gobbling drops': the jetting-dripping transition in flows of polymer solutions," J. Fluid Mech. 636, 5-40 (2009).

[40] McKinley, G. H., V. Sharma, B. Keshavarz, E. Houze, P. Cotts, J. Moore, and M. Pottiger, "High deformation rate extensional rheometry of complex fluids," ICR 2012-16th International Congress on Rheology, Lisbon, Portugal, 2012.

[41] Greiciunas, E., J. Wong, I. Gorbatenko, J. Hall, M. C. T. Wilson, N. Kapur, O. G. Harlen, D. Vadillo, and P. Threlfall-Holmes, "Design and operation of a Rayleigh Ohnesorge jetting extensional rheometer (ROJER) to study extensional properties of low viscosity polymer solutions," J. Rheol. 61, 467-476 (2017). 
[42] Graessley, W. W., "Polymer-chain dimensions and the dependence of viscoelastic properties on concentration, molecular-weight and solvent power," Polymer 21, 258-262 (1980).

[43] Larson, R. G., The Structure and Rheology of Complex Fluids (Oxford University, Oxford, 1999).

[44] Brandrup, J., and E. Immergut, Polymer Handbook, 4th ed. (John Wiley \& Sons, New York, 1999).

[45] McKinley, G. H., and A. Tripathi, "How to extract the Newtonian viscosity from capillary breakup measurements in a filament rheometer," J. Rheol. 44, 653-670 (2000).

[46] Slobozhanin, L. A., and J. M. Perales, "Stability of liquid bridges between equal disks in an axial gravity-field," Phys. Fluids A Fluid Dyn. 5, 1305-1314 (1993).

[47] Canny, J., “A computational approach to edge-detection," IEEE. Trans. Pattern Anal. Mach. Intell. 8, 679-698 (1986).

[48] Marr, D., and E. Hildreth, "Theory of edge-detection," Proc. R. Soc. Lond. B 207, 187-217 (1980).

[49] Spiegelberg, S. H., D. C. Ables, and G. H. McKinley, "The role of endeffects on measurements of extensional viscosity in filament stretching rheometers," J. Non-Newtonian Fluid Mech. 64, 229-267 (1996).

[50] Wagner, C., Y. Amarouchene, D. Bonn, and J. Eggers, "Droplet detachment and satellite bead formation in viscoelastic fluids," Phys. Rev. Lett. 95, 164504 (2005).

[51] Oliveira, M. S. N., and G. H. McKinley, "Iterated stretching and multiple beads-on-a-string phenomena in dilute solutions of highly extensible flexible polymers," Phys. Fluids 17, 071704 (2005).

[52] Sattler, R., C. Wagner, and R. J. Eggers, "Blistering pattern and formation if nanofibers in capillary thinning of polymer solutions," Phys. Rev. Lett. 100, 164502 (2008).

[53] Hinkley, D. V., "On ratio of 2 correlated normal random variables," Biometrika 56, 635-639 (1969).

[54] Hayya, J., D. Armstrong, and N. Gressis, "Ratio of 2 normally distributed variables," Manage. Sci. A 21, 1338-1341 (1975).

[55] Eggers, J., and T. F. Dupont, "Drop formation in a one-dimensional approximation of the Navier-Stokes equation," J. Fluid Mech. 262, 205-221 (1994).

[56] Li, J., and M. A. Fontelos, "Drop dynamics on the beads-on-string structure for viscoelastic jets: A numerical study," Phys. Fluids 15, 922-937 (2003).

[57] Fontelos, M. A., and J. Li, "On the evolution and rupture of filaments in Giesekus and FENE models," J. Non-Newtonian Fluid Mech. 118, 1-16 (2004).

[58] Bird, R., C. Curtiss, R. Armstrong, and O. Hassager, Dynamics of Polymeric Liquids. Volume 2: Kinetic Theory (John Wiley \& Sons, New York, 1987).
[59] McKinley, G. H., "Visco-elasto-capillary thinning and break-up of complex fluids," Rheol. Rev. 3, 1-48 (2005).

[60] Papageorgiou, D. T., "On the breakup of viscous liquid threads," Phys. Fluids 7, 1529-1544 (1995).

[61] Bhat, P. P., S. Appathurai, M. T. Harris, and O. A. Basaran, "On self-similarity in the drop-filament corner region formed during pinch-off of viscoelastic fluid threads," Phys. Fluids 24, 083101 (2012).

[62] Clanet, C., and J. C. Lasheras, "Transition from dripping to jetting," J. Fluid Mech. 383, 307-326 (1999).

[63] H. Sachsenheimer, H. B. B., and D. N. Willenbacher, "Determination of axial forces during the capillary breakup of liquid filaments with the tilted caber method," Rheol. Acta 51, 909-923 (2012).

[64] Prabhakar, R., J. R. Prakash, and T. Sridhar, "Effect of configurationdependent intramolecular hydrodynamic interaction on elastocapillary thinning and breakup of filaments of dilute polymer solutions," J. Rheol. 50, 925-947 (2006).

[65] Prabhakar, R., S. Gadkari, T. Gopesh, and M. Shaw, "Influence of stretching induced self-concentration and self-dilution on coil-stretch hysteresis and capillary thinning of unentangled polymer solutions," J. Rheol. 10, 345-366 (2016).

[66] Prabhakar, R., C. Sasmal, D. Nguyen, T. Sridhar, and J. R. Prakash, "Effect of stretching-induced changes in hydrodynamic screening on coil-stretch hysteresis of unentangled polymer solutions," Phys. Rev. Fluids 2, 011301 (2017).

[67] McIlroy, C., O. G. Harlen, and N. F. Morrison, "Modelling the jetting of dilute polymer solutions in drop-on-demand inkjet printing," J. Non-Newtonian Fluid Mech. 201, 17-28 (2013).

[68] Apelian, M. R., R. Armstrong, and R. A. Brown, "Impact of the constitutive equation and singularity on the calculation of stick slip-flow the modified upper-convected Maxwell model (mucm)," J. NonNewtonian Fluid Mech. 27, 299-321 (1988).

[69] Anna, S. L., and G. H. McKinley, "Elasto-capillary thinning and breakup of model elastic liquids," J. Rheol. 45, 115-138 (2001).

[70] Vadillo, D. C., M. Tembely, N. F. Morrison, O. G. Harlen, M. R. Mackley, and A. Soucemarianadin, "The matching of polymer solution fast filament stretching, relaxation, and break up experimental results with $1 \mathrm{~d}$ and $2 \mathrm{~d}$ numerical viscoelastic simulation," J. Rheol. 56, 1491-1516 (2012b).

[71] Szabo, P., G. H. McKinley, and C. Clasen, "Constant force extensional rheometry of polymer solutions," J. Non-Newtonian Fluid Mech. 169, 26-41 (2012).

[72] Keunings, R., "On the Peterlin approximation for finitely extensible dumbbells," J. Non-Newtonian Fluid Mech. 68, 85-100 (1997). 